\title{
Fraud Beliefs Following the 2020 U.S. Presidential Election: A Belief Systems Analysis
}

\author{
Rotem Botvinik-Nezer ${ }^{1}$, Matt Jones ${ }^{2}$, Tor D. Wager $^{1 *}$ \\ ${ }^{1}$ Department of Psychological and Brain Sciences, Dartmouth College; Hanover, NH, USA \\ ${ }^{2}$ Department of Psychology and Neuroscience and Institute of Cognitive Science, University \\ of Colorado Boulder; Boulder, CO, USA \\ *Corresponding author. Email: Tor.D.Wager@dartmouth.edu.
}

\begin{abstract}
Beliefs that the 2020 Presidential election was fraudulent are prevalent across the U.S. despite substantial contradictory evidence. We surveyed 1642 Americans during the U.S. Presidential vote count on November 4-5, assessing fraud beliefs and presenting hypothetical election outcomes before key states were decided. Participants' fraud beliefs increased when their preferred candidate lost and decreased when he won, and this effect scaled with preference strength. A Bayesian model accounts for this bias as reflecting a rational attribution process operating on biased prior beliefs about the true election winner and beneficiary of fraud. Our findings suggest that a systems approach targeting multiple beliefs simultaneously may be more fruitful in combating false beliefs than direct "debunking" attempts.
\end{abstract}




\section{Main Text}

On January 6, 2021, the U.S. congress assembled in the Capitol for the electoral vote count that would formalize Joe Biden's election as the new President. Outside the Capitol building, thousands of Americans participated in a riot based in part on claims that the election was fraudulent. Many of them broke into the Capitol building, endangering members of Congress and forcing an evacuation. These events were preceded, and followed, by widespread attempts to debunk beliefs in election fraud with substantial contradicting evidence. These attempts largely failed.

What are the psychological underpinnings of such beliefs? Studies have demonstrated that beliefs can be updated in a biased manner, deviating from rational (i.e., normative probabilistic) inference. For example, people update their beliefs more in response to confirmatory compared to disconfirmatory information (a 'confirmation bias'; $(1,2)$ ), and to desired compared to undesired information (a 'desirability bias'; (3-5)). Whereas this previous work has focused on updating of single beliefs, here we examine whether belief in election fraud can be better understood within a system of other beliefs that interact with and support it.

In a belief systems perspective, multiple beliefs combine or compete to explain observed evidence $(6,7)$. This perspective offers a potential mechanism for biased belief updating: When confronted with an undesired outcome (losing an election), people can attribute it to an alternative causal explanation (fraud) as a means to protect desired beliefs (that their preferred candidate was the true winner). Alternatively, the desirability effect could be a product of rational updating of multiple interacting beliefs $(6-10)$. According to this view, fraud beliefs result from abductive inferences (or 'inference to best explanation') about which of two potential causal explanations (true votes or fraud) is the most likely explanation for the outcome. From a Bayesian perspective, increasing fraud belief is normative (i.e., rational) to the extent that it explains away an outcome inconsistent with other prior beliefs. That is, if a candidate is expected to win but loses, the posterior probability of alternative explanations such as fraud increases. If both sides expect their candidate to win, then normative updating could explain the selective increase in fraud when one's preferred candidate loses. These two possibilities - inference biased toward desired conclusions and unbiased inference from skewed prior beliefs - point to different strategies for combating fraud beliefs, both of which go beyond straightforward attempts to debunk them with counterevidence.

We tested these ideas in an ecological, high stakes setting. We surveyed a large, representative online sample of 1642 Americans during the 2020 U.S. presidential election on November 4-5, while the winner was not yet determined and votes were being counted in key states. The sample included participants from each of the 50 U.S. states and the District of Columbia, with age ranging from 18 to 84 years (Fig. S1). Participants reported their preferences for President (Biden or Trump) and strength of preference, prior beliefs about the probability of a win by their preferred candidate (prior win belief), and the probability that election fraud would play a significant role in the outcome (prior fraud belief; see Table S1 for all survey questions). We then showed each participant a hypothetical map representing the winner in each state and the electoral votes for each candidate, randomized to indicate either a Republican (Trump) or Democratic (Biden) victory (Fig. 1A-B). Participants reported the probability that fraud played a significant role in the outcome shown on the hypothetical map (i.e., their posterior fraud belief). Based on the belief systems perspective, we predicted that even in the absence of any direct information about fraud, people would update their fraud beliefs as an alternative explanation for an undesired and/or disconfirmatory outcome. Pre-registered hypotheses and analysis plan (after data collection but prior to inspection of the beliefs data) are available from the Open Science Framework (https://osf.io/kucsw; for further details see Materials and Methods). 
A

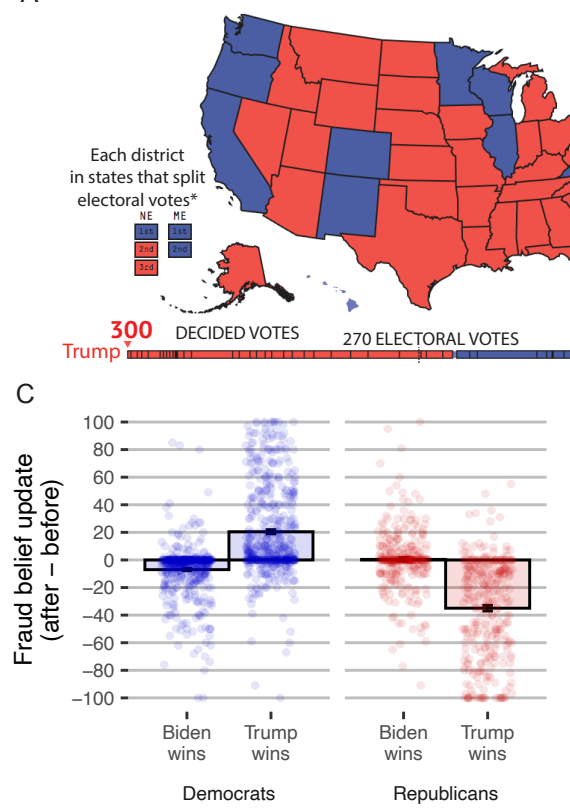

B

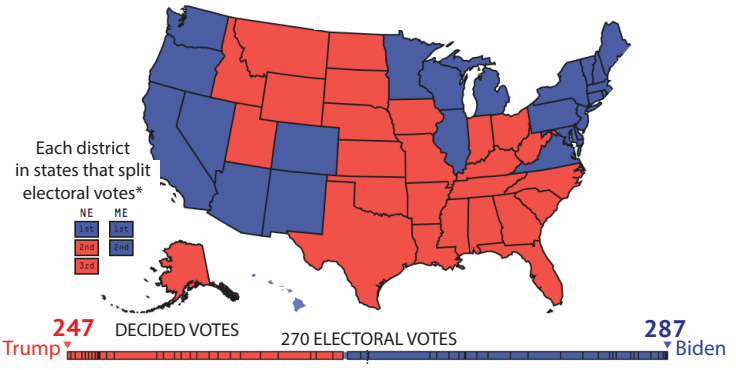

D
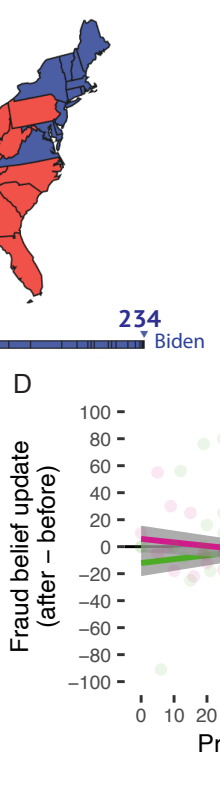

E

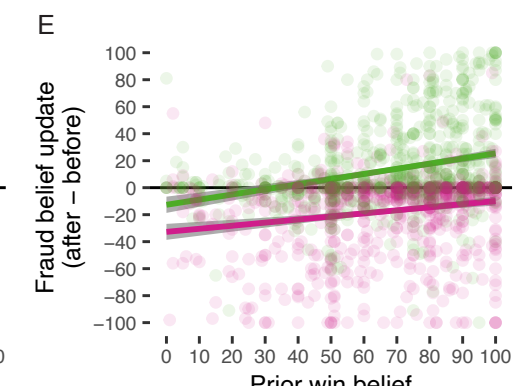

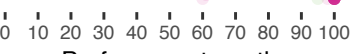
Preference strength

Map type

Undesired (loss)

Desired (win)

Fig. 1. Empirical fraud belief update given hypothetical election outcome maps.

Participants were randomly presented with either (A) a map showing a Republican win (Trump, red) or (B) a map showing a Democratic win (Biden, blue). The bottom scale shows the distribution of electoral votes (the party receiving more than 270 electoral votes wins the Presidential election). Maps were created by flipping four out of the six states that were considered critical at the time of the survey with votes still being counted, using the election tool provided by the fivethirtyeight.com website: https://projects.fivethirtyeight.com/trump-biden-election-map/. (C) Fraud belief updates following the hypothetical maps are shown separately for Democrats and Republicans, for each scenario. Higher values indicate stronger belief in fraud. Points represent single participants, and error bars represent

standard error of the mean across participants. The same plot with prior and posterior fraud beliefs presented separately is shown in Fig. S3A, emphasizing the difference in prior fraud beliefs between the two partisan groups. (D-E) Results are aggregated across the partisan groups and the fraud belief update is shown as a function of (D) the strength of preference towards the preferred candidate (neutral $=0$ ), and $(\mathbf{E})$ the prior win belief (probability of the preferred candidate's win; equal probability $=50$ ). Points represent single participants. Data are analyzed separately for Democratic and Republican participants in the Extended Results (see Supplementary Materials).

Participants' preferences for one candidate were strong across the sample, with $52.7 \%$ indicating the strongest possible preference and only $4.8 \%$ indicating a strength of preference in the lower half of the scale (Biden supporters [Democrats]: $M=89.6, S D=18.4$ on a 100-point scale; Trump supporters [Republicans]: $M=85.8, S D=19.6$; two sample t-test: $t_{(1640)}=3.95, p<.001$; Fig. S2A). Most participants believed their preferred candidate would win the election, with stronger beliefs for Biden than Trump supporters (estimated probability of preferred candidate's win: Democrats: $M=77.5 \%, S D=15.5 \%$; Republicans: $M=50.3 \%, S D=26.4 \%$; Welch two sample t-test: $t_{(861.49)}=23.28, p<.001$; Fig. S2B). Overall, $75.6 \%$ of participants believed their candidate had a greater than $50 \%$ chance of winning. Prior to presentation of the hypothetical election outcome, participants' fraud beliefs were moderate across the sample, and higher for Republicans than Democrats (Democrats: $M=19.9 \%, S D=24.4 \%$; Republicans: $M=63.9 \%, S D=30.7 \%$; Welch two sample t-test: $t_{(1061.6)}=-30.11, p<.001$; Fig. S2C). This difference scaled with preference: the correlation between prior fraud beliefs and preference strength was significantly 
positive for Republicans (Pearson $r=0.35 ; p<.001$ ) but significantly negative for Democrats (Pearson $r=-0.22 ; p<.001$ ).

We observed strong evidence for partisan preference effects in fraud belief updating. Participants' fraud beliefs increased substantially after a preferred-candidate loss (one sample t-test: $M=12.97$, $\left.S D=31.11, t_{(823)}=11.97, p<.001\right)$ and decreased substantially after a preferred-candidate win (one sample t-test: $M=-17.35, S D=29.41, t_{(817)}=-16.87, p<.001$; Fig. $1 C$ ). The directional update was weaker in the "Biden wins" scenario, likely for two reasons. First, many Republicans had maximal prior fraud beliefs that could not increase given a loss, creating a ceiling effect; conversely, many Democrats had minimal prior fraud beliefs that could not decrease given a win (Fig S3A). Second, a Biden win was the more probable scenario based on the participants' prior win beliefs. According to our Bayesian model described below, rational updating of fraud beliefs depends on the degree to which the observed outcome violates prior expectations.

These directional updates of fraud beliefs were significantly associated with the strength of (a) participants' preference for one candidate and (b) prior win beliefs (each controlling for the other in a multiple regression model; Fig. 1D-E). Following a preferred-candidate loss, increases in fraud beliefs were greater for those with stronger partisan preferences $\left(\beta=0.10, t_{(800)}=3.20, p=.001\right)$, and when participants were more surprised by the outcome, i.e., when deviations from prior expectations were largest $\left(\beta=0.16, t_{(800)}=4.31, p<.001\right.$; Table S2). Following a preferredcandidate win, fraud beliefs decreased more strongly with stronger preferences $\left(\beta=-0.11, t_{(799)}=\right.$ $-5.05, p<.001)$, but the update was not significantly related to participants' prior win belief $(\beta=$ $0.03, t_{(799)}=-1.11, p=.265$; Table S3). Additional details about covariates, robustness across model variants, and effects for Democrats and Republicans separately can be found in the Supplementary Materials and Fig. S3B.

These results indicate that in a high-stakes ecological setting, people update their fraud beliefs substantially in the absence of direct evidence for or against fraud. These updates were strongly related to preferences about the election winner, consistent with other types of desirability bias (see (4) for a review) and motivated reasoning (3), but also to winner expectations. Overall, we observed three effects: (a) Participants believed in fraud less when they liked the election outcome, and more when they did not (a "direction desirability effect"), and these updates were larger (b) when the preference was stronger (a "magnitude desirability effect"), and (c) when the outcome was less expected, at least for the loss scenario (a "surprise effect"). These effects generalized to members of both political parties (Supplementary Materials).

The surprise effect is compatible with Bayesian abductive inference: the less likely a result is under a fraudless election, the more strongly it indicates fraud. On the other hand, the two desirability effects seem to challenge the Bayesian account, because preference per se has no role in rational belief updating; however, they might emerge from rational belief updating if fraud inferences depend on other beliefs that may themselves be biased ( 8 ).

To investigate whether all three findings are jointly compatible with Bayesian abductive inference, we formulated a Bayesian model with the potential to explain all three of these effects in a simple mechanistic framework, and to make quantitative predictions about the dynamics of belief updating at both the group and individual-participant level (the model was not included in our preregistration). The model formalizes the abductive inference concept by describing a rational observer of an election outcome with two candidates (A and B) jointly determined by three binary causal variables (Fig. 2A): the true votes ( $V$ : election outcome given no fraud), presence of 
substantial fraud that could affect the outcome $(F)$, and the side perpetrating any such fraud $(C)$.
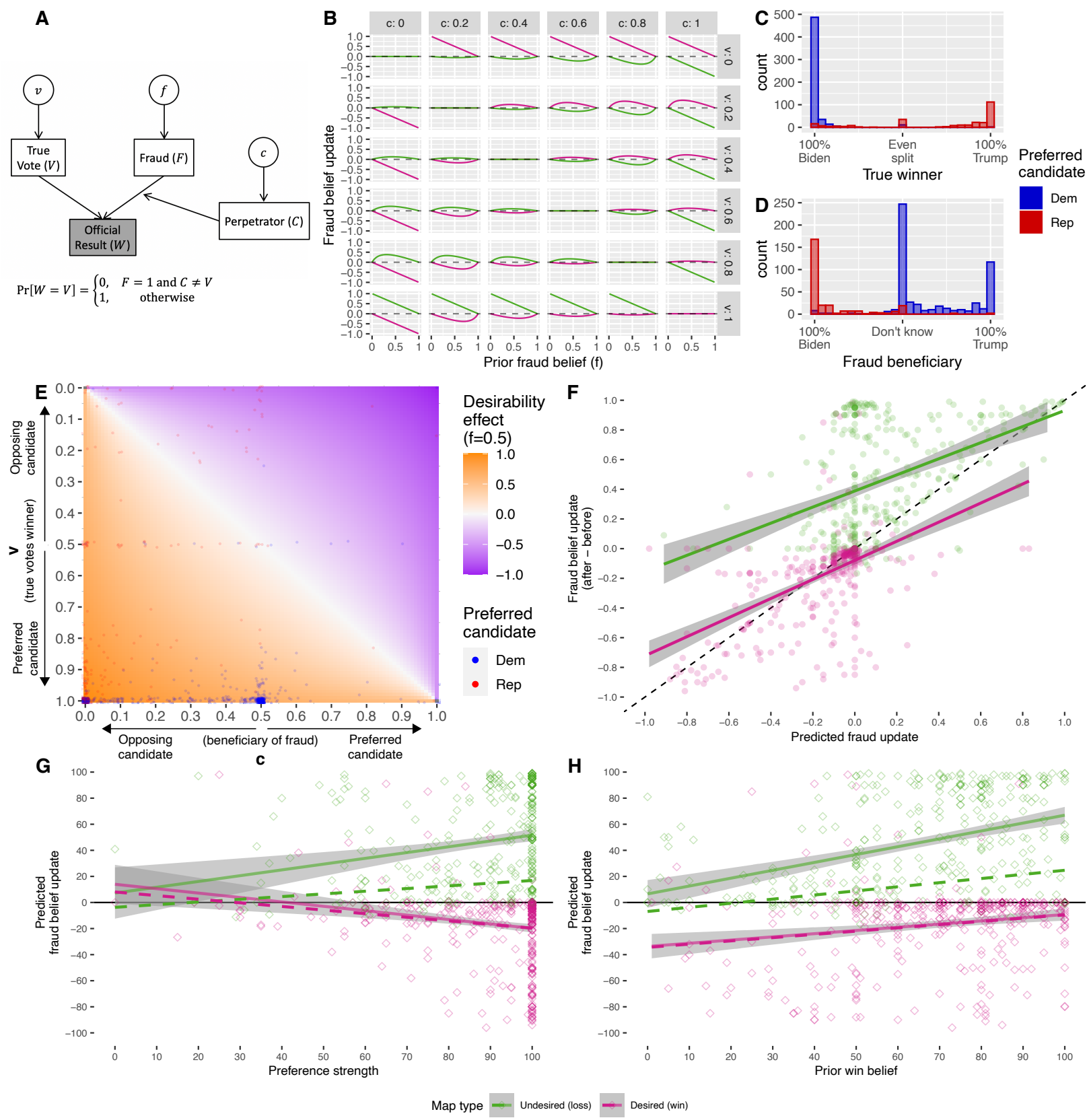

Fig. 2. Bayesian model, priors and predictions.

A Bayesian model provides quantitative predictions about the posterior fraud belief in a belief systems framework. (A) The generative model. The observer begins with a prior belief state comprising the probability that candidate A (the participant's preferred candidate, without loss of generality) will win the true vote $(v)$, the probability of substantial fraud $(f)$, and the probability that fraud (if present) favors candidate A $(c)$. After observing the official election outcome, these beliefs are updated according to Bayes' rule (see Materials and Methods). (B) The Bayesian model's predicted fraud belief updates across the parameter space defined by $f, v$ and $c$. Green and pink: preferredcandidate loss and win, respectively. (C-D) Histograms of participants' empirically measured prior beliefs regarding

(C) the true (fraudless) winner and (D) the candidate benefiting more from fraud. (E) Heat map of the model's predicted desirability effect (calculated as the posterior fraud belief for loss minus posterior fraud belief for win), as

a function of $v$ and $c$, when prior fraud belief $f=0.5$. In the areas shaded orange, fraud beliefs increase for an undesired loss and decrease for a desired win (a direction desirability effect). The points represent single participants based on empirically measured priors (points are jittered slightly for visibility). (F) Predicted fraud belief updates 
from the model, based on empirically measured priors, are significantly correlated with empirically observed updates $(r=0.62)$. (G-H) Reproducing the empirical results from Fig. 1D-E, the Bayesian model-based predictions of the fraud belief update for each participant are presented as a function of $(\mathbf{G})$ the empirically measured prior subjective probability of the preferred candidate's win and $(\mathbf{H})$ the empirical strength of preference towards the preferred candidate. For comparison, dashed lines show the observed empirical patterns (Fig. 1D-E)

The observer's prior belief constitutes a probability distribution over these variables, taken to be independent with subjective probabilities $v$, the probability that candidate A will win the most true votes $\mathrm{V} ; f$, the probability of substantial fraud $\mathrm{F}$; and $c$, the probability that fraud (if present) favors candidate A. The candidate winning the true vote will win the election unless substantial fraud exists and favors the other candidate, in which case the latter would win. The model provides a quantitative prediction for the posterior fraud belief conditional on a win $(W)$ by candidate A or by candidate B (see Materials and Methods for full derivation):

$\operatorname{Pr}[F=1 \mid W=A]=\frac{f c}{v+f(c-v)}$
$\operatorname{Pr}[F=1 \mid W=B]=\frac{f(1-c)}{1-v+f(v-c)}$

Defining A as the preferred candidate for convenience, and without loss of generality, the model predicts that a rational agent observing a win by their preferred candidate will decrease fraud beliefs if $v>c$ and increase fraud beliefs if $c>v$, and vice versa for a win by the dispreferred candidate (Fig 2B). Thus, the model posits that desirability effects in fraud beliefs arise from two interacting beliefs: The prior belief that one's preferred candidate is more likely to win (higher $v$ ) and the prior belief that the opponent is more likely to be the beneficiary of fraud (lower $c$ ). This exemplifies a computational principle underlying abductive inference: Fraud belief will be increased to the degree that it is a better (more likely) explanation for candidate A's win (or loss) than is winning (or losing) the true vote. Conversely, if fraud is a less likely explanation for the outcome than is the true vote, belief in fraud will decrease.

To test whether the model fits the observed data, we conducted a follow-up survey collected from $N=937$ of the original participants shortly before the inauguration of Joe Biden (January 19-20, 2021, about 11 weeks after the original survey; the follow-up survey was not included in our preregistration). $N=828$ participants remained after exclusions (see Materials and Methods). Participants' fraud beliefs in the follow-up survey were consistent with their ratings from the original survey after the hypothetical outcomes: For participants in the 'Biden win' scenario (the official outcome), ratings of the probability of fraud in favor of Biden 11 weeks post-election were correlated with the pre-election fraud ratings given the hypothetical map $(N=424$, Spearman's $\rho$ $=0.66, p<.001$ ). In the follow-up survey, participants self-reported $v$ and $c$ (see Table $\mathrm{S} 4$ for full survey questions). As illustrated in Fig. 2C-E (and Fig. S4A-B), almost all participants from both parties believed that (a) their candidate would have been the true winner in the absence of fraud $(v \approx 1)$ and $(b)$ if there was fraud, it favored the dispreferred candidate $(c \leq 0.5)$. Nearly all participants occupied a portion of the $(v, c)$ parameter space that would lead to a strong direction desirability effect, as we observed empirically (for extended details regarding the divergent beliefs of the two partisan groups see the Supplementary Materials).

Moreover, the predicted desirability effect gets stronger as $v$ increases and $c$ decreases (Fig. 2E). Because the empirical bias was positively related to partisan preference in the original survey (the magnitude desirability effect, Fig. 1D), we tested whether empirical ratings of $c$ and $v$ were correlated with preference strength in the original survey. Indeed, those with stronger preference 
also believed more strongly that (a) their preferred candidate would have been the true winner (Republicans: $r=0.58, N=270, p<.001$; Democrats: $r=0.24, N=558, p<.001$ ), and (b) fraud favors the opposing side (Republicans: $r=-0.51, N=270, p<.001$; Democrats: $r=-0.22, N=$ $558, p<.001)$. Participants with the strongest preferences occupied the portion of the $(v, c)$ parameter space where the model predicts the largest desirability effect (Fig. S4C-D), explaining the magnitude desirability effect we observed empirically.

The model also provided quantitative predictions of individual participants' fraud beliefs that matched the empirical data well. The model was fit with no free parameters. Participants selfreported $v$ and $c$ in the follow-up survey were used to predict participant-level fraud belief updates in the original survey. ${ }^{1}$ The correlation between the predicted and observed fraud belief updates across $N=729$ participants with defined predicted values was $r=0.62(p<.001 ; R M S E=0.381$; Fig. 2F). The empirical desirability and surprise effects were also both predicted by the model (Fig. 2G-H). The model fit the data better for the win scenario $(r=0.53, N=399, p<.001, R M S E$ $=0.266)$ than the loss scenario $(r=0.44, N=330, p<.001, R M S E=0.486)$, for which the predicted updates were mostly larger than observed.

In sum, the Bayesian model explains both the two desirability effects and the surprise effect revealed in the empirical data. Importantly, it explains the strong partisan biases in fraud beliefs we observed as a function of interacting prior beliefs operating on a rational Bayesian attribution and belief-updating process. While Democrats expect Biden to win the true votes and fraud (if any) to favor Trump, Republicans expect Trump to win the true votes and fraud to favor Biden (Fig. 2C-D). Clearly, both groups cannot be right. A rational actor operating on these biased prior beliefs will show an asymmetric updating of fraud belief, in favor of one's preferred candidate and in the absence of direct evidence for or against fraud.

The model was not intended as a complete model of belief formation, and its predictions did not capture all features of the empirical data. For example, the model does not include selective exposure to information, which can operate to induce biases beyond the mechanisms we explored here (11-13). Two main types of mismatch between model and data are worth noting (Fig. S5): (a) In the loss scenario, the model predicted larger increases in fraud beliefs than empirically observed, mainly for the Democratic participants. This might be because the $v$ values used for the model's predictions were taken from the follow-up survey, after the Democratic candidate won the official election, and therefore may have been higher for these participants than their unobserved $v$ during the original survey. (b) In the win scenario, participants with very high prior fraud belief $(f \approx 1)$ updated more than the model predicted. A Bayesian account predicts resistance to new evidence when priors are very strong. Here, people may have updated more because they reason imperfectly about probabilities, or because they may report extreme beliefs partly as a statement of allegiance ('expressive responding' (14), but see (15)).

The model also predicts an election's ability to accomplish its primary purpose, in the form of assessing people's willingness to alter their beliefs in the true winner, $v$ (Fig. S6). The posterior belief in the model given the official outcome $(W)$ is:

$\operatorname{Pr}[V=A \mid W=A]=\frac{v(1-f)+v f c}{v+f(c-v)}$

\footnotetext{
${ }^{1}$ One caveat is that, since $v$ and $c$ were taken from the follow-up rather than the original survey, they are better interpreted as posterior rather than prior beliefs.
} 
$\operatorname{Pr}[V=A \mid W=B]=\frac{v f(1-c)}{1-v+f(v-c)}$

Based on the empirically obtained priors $f, v$ and $c$ in our sample, our model predicts that after a loss by their preferred candidate, $83.6 \%$ of participants would increase their belief in fraud more than they would decrease their belief that their true candidate is the rightful winner. For $67.5 \%$ of the participants, more than $90 \%$ of the overall update will be to increase fraud beliefs rather than change belief in the true winner. To account for the previously described issue that reports of extreme beliefs (probability of 0 or 1) may be partially heuristic and unlikely to reflect actual complete certainty, we re-estimated this proportion after regularizing $f, v$ and $c$ values to be between 0.05 to 0.95 . Under this change, the model predicts that $51.8 \%$ of our participants will increase their fraud belief more than they will decrease their true vote belief following a loss. For $19.3 \%$, more than $90 \%$ of the update will be attributed to the fraud rather than true vote belief. These results suggest that a significant portion of the population might be "election-proof", in the sense that an official loss by their favored candidate will not change their beliefs in the rightful winner, and they will instead attribute the loss almost entirely to fraud. This is supported by both our follow-up data and national polls. For example, 39.3\% of Republicans in our sample were still certain that Trump was the true winner weeks after Biden was declared as the official winner, and national polls since the 2020 election and months after consistently revealed that about one third of Americans continue to believe that Biden won only due to fraud (https://www.monmouth.edu/polling-institute/documents/monmouthpoll_us_062121.pdf/, https://cdn.cnn.com/cnn/2021/images/04/30/rel3e.-.voting.and.elections.pdf).

Together, these findings illustrate how public trust in elections depends on dynamic interactions among several beliefs $(16,17)$, and how specific combinations of beliefs can prevent democratic elections from achieving their primary purpose. Beliefs in election fraud have played a substantial role in undermining democratic governments worldwide (18), and have grown and remained strikingly prevalent in the U.S. $(17,19)$. Belief in fraud undermines both motivation to vote and acceptance of election results, which bears directly on the viability of an elected government. Previous studies have found a consistent "winner effect", where people are more likely to trust election outcomes when they win compared to when they lose $(20,21)$, similar to the effect we found here.

Importantly, our findings and model suggest that fraud beliefs do not exist in isolation, but are part of a system of interacting beliefs. They can emerge in the absence of a biased or irrational updating process, depending on the strength of two other prior beliefs: (a) that one's preferred candidate truly has the people's vote ( $V$ in our model; Fig $2 \mathrm{C}$ ) and (b) that fraud, however prevalent, will favor the opponent ( $C$ in the model; Fig 2D). These prior beliefs determine how credit for observed evidence is distributed across latent causes. Specifically, the attribution of an election outcome to a legitimate win (the "will of the people") versus a fraudulent one depends on which cause provides the better (more likely) explanation for the outcome. Evidence that could guide these beliefs separately is difficult to obtain at best: Direct evidence on the true election winner $V$, in particular, requires a fraudless election. Rhetoric that increases belief in fraud can thus create a 'short circuit': An election outcome $(W)$ might be intended to provide evidence for $V$, but if one side is believed to be cheating disproportionately $(C)$, then observing $W$ serves to update fraud beliefs ( $F$ and $C$ ) rather than affecting the intended belief $V$. The right combination of prior beliefs can even create a situation where belief in fraud becomes nearly unfalsifiable. Many participants in our study reported prior beliefs in a range that creates the conditions for self-perpetuating fraud beliefs that persist independent of, and are resistant to, direct evidence about fraud. 
This framework has implications for efforts to counteract fraud beliefs in the U.S. and beyond. Such efforts typically focus on directly "debunking" them, i.e., providing evidence against fraud. But these debunking efforts have largely failed, and fraud beliefs' prevalence has not decreased significantly (22). Our model affords new ways of thinking about how to decrease false beliefs. Specifically, it suggests that debunking attempts must broaden to include the broader system of interacting beliefs. To reduce belief in election fraud, in addition to (a) directly targeting fraud beliefs with evidence that fraud is very rare (decreasing $f$ in our model), it might be advantageous to simultaneously target beliefs that (b) Americans have diverse preferences, and one's dispreferred candidate truly is supported by many (decreasing $v$ ), and (c) when fraud does occur its benefits are distributed across both candidates (increasing $c$ ). Real-world political campaigns already target multiple beliefs in this fashion to varying degrees, though perhaps without this explicit formulation. For example, the Trump campaign simultaneously targeted these same three beliefs before and during the 2020 election: (a) "It's rigged": Election fraud is rampant (increase f); (b) "We're winning": Trump is popular and enjoys the true support of most Americans (increase $v$ for Trump); and (c) "The Democrats are crooked": Fraud overwhelmingly favors the opposition (decrease $c$ ). Although these messages may initially seem unrelated, they all fit together within the framework we describe here, as a coordinated strategy to create evidence-resistant beliefs about the true winner.

Our findings indicate that the apparent desirability bias is a result of biased prior beliefs, rather than biased inference. The sources of the prior beliefs that are so critical here include many social, technological, and psychological processes beyond the scope of our model and study. For example, social media algorithms have tended to select sources of evidence that agree with individuals' prior preferences, creating "filter bubbles" and "echo chambers" $(12,23)$ and social media use and polarization are thought to be mutually reinforcing (13)- direct causal links between prior preferences and evidence exposure. Likewise, individuals often want to hold certain beliefs, and often select information that reinforces them $(11,24,25)$. Prior beliefs are also shaped powerfully by social norms and group identity $(26,27)$. Our model does not attempt to explain these varied and powerful processes, but our hope is that it provides a way to help understand the dynamics of their influence.

More broadly, our findings highlight the importance of a systems perspective in understanding human beliefs across domains, and in particular the critical role of attribution. The dynamics we observed here may be at play in multiple areas crucial for human wellbeing, including beliefs about the self and others that shape individual mental health (28), beliefs in the efficacy and safety of vaccines (29), beliefs in the need for action to address climate change $(30,31)$, and more. In each of these domains, evidence must be attributed across multiple latent causes, which shapes the dynamics of which beliefs are reinforced by observed evidence.

Our findings and model do not preclude the existence of other (Bayesian or non-Bayesian) mechanisms underlying bias towards desired beliefs. For example, desires may influence the degree of attention paid to particular pieces of evidence (e.g., one can ignore incompatible information), how evidence from different sources is weighted, and how much mental work and imagination are devoted to formulating alternative causal explanations for evidence that challenges cherished beliefs. These possibilities illustrate some of the variables that must be systematically investigated before a more complete model of human belief formation can be developed. All in all, our findings and model suggest that interconnected beliefs should be considered, studied, and targeted simultaneously in order to better understand their dynamics and to combat false beliefs more effectively. 


\section{Materials and Methods}

\section{Participants}

A large nationally representative online sample of 1760 American citizens was collected from Amazon's Mechanical Turk (MTurk; Amazon, Seattle, WA) via Cloud Research (32). The sample size was chosen based on available funds aiming for a large sample across the U.S. Participants were 18 years old or older and provided their consent for participation online prior to accessing the survey questions. The study was approved by Dartmouth College's Committee for the Protection of Human Subjects.

Thirty-two participants were excluded due to failure to correctly answer a simple attention check included in the survey and one due to not meeting the participation requirement of American citizenship or permanent residency. Eighty-five additional participants were excluded from analyses because they chose "Other" for their preferred Presidential candidate (and not "Donald Trump" or "Joe Biden"). Final analyses included 1642 participants, of which 1032 preferred Joe Biden and 610 preferred Donald Trump. Partisan affiliation was generally, but not perfectly, aligned with the preferred candidate (Fig. S1A). We use Democrat and Republican to refer to participants according to their preferred candidates (Biden and Trump, respectively). The sample included 1 to 138 participants from each of the 50 U.S. states and the District of Columbia (Fig. S1B). The age of participants ranged from 18 to 84 years old $(M=41.6, S D=13.1$ years $)$ and Republicans were older compared to Democrats (Democrats: $M=39.9, S D=12.8$ years; Republicans: $M=44.5, S D=13.1$ years; Fig. S1C).

\section{Materials and Procedures}

Each participant completed a survey distributed on the Qualtrics platform (Qualtrics, Provo, UT). The U.S. election took place on November 3rd, 2020. The survey was available online from November 4th at 8 pm until November 5th at 10:30 am Eastern Time. During that time, votes were still being counted and there was no conclusive winner. Six states were considered critical "swing states" with no clear winner and votes still being counted: Nevada, Arizona, Michigan, Pennsylvania, North Carolina, and Georgia. We created two hypothetical maps that differed by flipping four of these six states: Nevada, Arizona, Michigan and Pennsylvania, using the election tool provided by fivethirtyeight.com: https://projects.fivethirtyeight.com/trump-biden-electionmap/. Each map provided a graphical representation of the states' final results (red for Republican victory and blue for Democrat victory), sum of electoral votes for Republicans and Democrats and the winner: one map with a Republican (Donald Trump) win and one with a Democrat (Joe Biden) win (Fig. 1A-B).

We collected participants' demographic information (age and state of residency), political affiliation (Republican, Democrat, Independent or Other), candidate preference (Donald Trump, Joe Biden or Other), preference strength (continuous scale from 0 [don't care either way] to 100 [extremely strong preference]), prior subjective probability of the preferred candidate's win (prior win belief; continuous scale from 0 [no chance] to 100 [definite win]) and prior subjective probability of fraud playing a significant role in the election outcome (prior fraud belief; continuous scale from 0 [not at all] to 100 [extremely]; see Table S1 for full description of the survey questions). Then, each participant was randomly presented with one of the two maps described above and were asked to indicate their belief about fraud affecting the election outcome if it were to turn out as in the map shown to them.

$\underline{\text { Analysis }}$ 


\section{Pre-registration}

We pre-registered our analysis plan and predictions on the Open Science Framework (https://osf.io/kucsw) after data collection but prior to inspection of beliefs-related data. We did examine some portions of the data, such as the number of participants supporting each candidate, as well as demographic information. These procedures did not inform us about the effects of interest. Rather, they were meant to monitor data quality and sample characteristics.

\section{Deviations from pre-registration}

We report a different multiple linear regression model than the one we pre-registered for the update of fraud belief, in order to simplify the model and interpretations. The differences between the two models, along with the results of the pre-registered model, which are in line with those of the revised one, are described in the Supplementary Materials.

The pre-registration was based mainly on the desirability bias hypothesis and focused on the empirical data from the original survey. After observing the data of the original survey, we developed the Bayesian model. A follow-up survey was then collected to test the model and expand the findings from the original survey.

\section{Code availability}

Our data and code are publicly shared (https://doi.org/10.5281/zenodo.5730631, release v1.0.0). All analyses were performed with R version 3.6.3 (https://www.r-project.org). For reproducibility, we used the checkpoint package, which installs all needed $\mathrm{R}$ packages as they were on a specific date. We set the date to June 30, 2021.

\section{Updating of fraud belief}

At the time of the pre-registration, we predicted, based on the desirability bias perspective and learning theories, that the update of fraud belief (posterior minus prior subjective probability of fraud playing a significant role in the election outcome) would be affected by two factors: (a) participants' preferences, such that fraud belief would increase when the hypothetical results were undesired, with a larger update for stronger preferences; and (b) participants' prior win belief, such that fraud belief will increase when the hypothetical results are less expected.

We tested our predictions with multiple linear regression predicting update of fraud belief as a function of the preference strength and the prior win belief, separately for the loss scenario (i.e., following a map in which the preferred candidate lost) and for the win scenario (i.e., following a map in which the preferred candidate won). The prior fraud belief, preferred candidate (Biden / Trump), participant's age, and order of survey submission during data collection were included in both models as covariates. The participant's state of residence was not included as it did not significantly reduce residual variance while substantially increasing model complexity. All numeric variables included in the model (dependent and independent) were z-scored. The same models were also tested separately for each partisan subgroup (preferred candidate = Democrat and preferred candidate $=$ Republican), where the preferred candidate covariate was omitted (see 
Supplementary Materials). Additionally, a one-sample two-sided t-test was used to test whether the mean change in fraud belief was significantly nonzero in each scenario.

\section{Bayesian model}

To test whether the general patterns of belief update we found can reflect rational inference from possibly biased prior beliefs, we formulated a Bayesian model that assumes participants attribute the election outcome to the combination of the true votes (i.e., election outcome given no fraud) and fraud (see Fig. 2A). The candidates are labeled A and B. Let $W, V \in\{\mathrm{A}, \mathrm{B}\}$ be the official and true winners, respectively. Let $F \in\{0,1\}$ indicate whether there was substantial fraud that could affect the outcome, and let $C \in\{\mathrm{A}, \mathrm{B}\}$ indicate which side committed fraud (only meaningful when $F=1)$. Therefore the official winner will match the true winner unless there was substantial fraud by the opposition $(F=1$ and $C \neq V)$ :

$\operatorname{Pr}[W=V]=\left\{\begin{array}{cc}0 & F=1 \text { and } C \neq V \\ 1 & \text { otherwise }\end{array}\right.$

We assume the participant has an independent prior on $V, F, C$, and denote $\operatorname{Pr}[V=\mathrm{A}]=v, \operatorname{Pr}[F$ $=1]=f, \operatorname{Pr}[C=\mathrm{A}]=c$. For convenience, we define $R \in\{\varnothing, \mathrm{A}, \mathrm{B}\}$ by $R=\emptyset$ when $F=0$ and $R$ $=C$ when $F=1$; that is, $R$ indicates who committed substantial fraud, with $\emptyset$ denoting no one. Then the joint prior on $V, R$ is given by

\begin{tabular}{c|c|c|}
\hline $\operatorname{Pr}[V, R]$ & $V=\mathrm{A}$ & $V=\mathrm{B}$ \\
\cline { 2 - 3 }$R=\varnothing$ & $v(1-f)$ & $(1-v)(1-f)$ \\
\cline { 2 - 3 }$R=\mathrm{A}$ & $v f c$ & $(1-v) f c$ \\
\cline { 2 - 3 }$R=\mathrm{B}$ & $v f(1-c)$ & $(1-v) f(1-c)$ \\
\cline { 2 - 3 }
\end{tabular}

The likelihoods, as described above, are given by

\begin{tabular}{c|c|c|}
$\operatorname{Pr}[W=\mathrm{A} \mid V, R]$ & $V=\mathrm{A}$ & $V=\mathrm{B}$ \\
\hline \multirow{2}{*}{$R=\varnothing$} & 1 & 0 \\
$R=\mathrm{A}$ & 1 & 1 \\
\cline { 2 - 3 }$R=\mathrm{B}$ & 0 & 0 \\
\cline { 2 - 3 } & &
\end{tabular}

The joint posteriors are then given by 


\begin{tabular}{c|c|c|}
$\operatorname{Pr}[V, R \mid W=\mathrm{A}]$ & \multicolumn{1}{c}{$V=\mathrm{A}$} & $V=\mathrm{B}$ \\
\cline { 2 - 3 }$R=\varnothing$ & $v(1-f) / Z_{\mathrm{A}}$ & 0 \\
\cline { 2 - 3 }$R=\mathrm{A}$ & $v f \mathcal{c} / Z_{\mathrm{A}}$ & $(1-v) f_{\mathcal{C}} / Z_{\mathrm{A}}$ \\
\cline { 2 - 3 }$R=\mathrm{B}$ & 0 & 0 \\
\cline { 2 - 3 } & &
\end{tabular}

and

\begin{tabular}{c|c|c|}
$\operatorname{Pr}[V, R \mid W=\mathrm{B}]$ & \multicolumn{1}{c}{$V=\mathrm{A}$} & $V=\mathrm{B}$ \\
\cline { 2 - 3 }$R=\varnothing$ & 0 & $(1-v)(1-f) / Z_{\mathrm{B}}$ \\
\cline { 2 - 3 }$R=\mathrm{A}$ & 0 & 0 \\
\cline { 2 - 3 }$R=\mathrm{B}$ & $v f(1-c) / Z_{\mathrm{B}}$ & $(1-v) f(1-c) / Z_{\mathrm{B}}$ \\
\cline { 2 - 3 } & &
\end{tabular}

with partition function

$$
\begin{aligned}
Z_{\mathrm{A}} & =\operatorname{Pr}[W=\mathrm{A}] \\
& =v(1-f)+v f c+(1-v) f c \\
& =v+f(c-v) \\
Z_{\mathrm{B}} & =\operatorname{Pr}[W=\mathrm{B}] \\
& =v f(1-c)+(1-v)(1-f)+(1-v) f(1-c) \\
& =1-v+f(v-c) .
\end{aligned}
$$

Marginalizing the joint posterior gives the posteriors on $F$ :

$$
\begin{aligned}
\operatorname{Pr}[F=1 \mid W=A]= & \frac{f c}{v+f(c-v)} \\
& =\frac{c}{c+(1-f)(v-c)} \operatorname{Pr}[F=1] \\
\operatorname{Pr}[F=1 \mid W=B]= & \frac{f(1-c)}{1-v+f(v-c)} \\
& =\frac{1-c}{1-c+(1-f)(c-v)} \operatorname{Pr}[F=1] .
\end{aligned}
$$

Therefore, Fraud belief increases if the official winner is more likely to be the cheater than to be the true winner (i.e., if $c>v$ for $W=\mathrm{A}$, and if $c<v$ for $W=\mathrm{B}$ ).

We also derived the posteriors for $V$, which are given by 
$\operatorname{Pr}[V=A \mid W=A]=\frac{v(1-f)+v f c}{v+f(c-v)}$

and

$\operatorname{Pr}[V=A \mid W=B]=\frac{v f(1-c)}{1-v+f(v-c)}$.

\section{Follow-up survey}

To assess the participants' beliefs about the true votes and who commits fraud, we collected a follow-up survey. The follow-up survey was again distributed on Qualtrics via Cloud Research and was available only to participants who completed the original survey. $N=937$ participants from the original sample completed the follow-up survey (nine additional ones failed the attention check) and were included in the analysis of the follow-up survey data. For the purpose of testing the Bayesian model and assessing the priors, we further excluded 44 participants who indicated "Other" as their preferred candidate during the original survey, 22 participants who indicated "Other" as their preferred candidate during the follow-up survey, and an additional 43 participants who changed their preferences between the surveys (all switched from preferring "Trump" to preferring "Biden"). We obtained the priors and applied the model on the remaining $N=828$ participants.

The survey was accessible between the evening of January 19, 2021 and the inauguration of Joe Biden (around 12 pm ET on January 20, 2021), and included questions about the participants' preferences (at the time of the follow-up survey completion and during the election), their beliefs about the winner of the election, their beliefs about fraud, and the activities they considered when answering the questions about fraud (see Table S4 for all survey questions). Participants' values for $v$ were computed from their answer to the question "Who would have won in a fraudless election?" along with their reported confidence in that answer (continuous scale 0 - 100). The confidence level was multiplied by -1 for participants who indicated the opponent candidate as the fraudless winner, and $v$ was set as the confidence level after rescaling to a range of $[0,1]$. For participants who answered "I don't know" with regard to the fraudless winner, we set their $v$ to 0.5 , indicating uncertainty. Participants' values for $c$ were computed from their answer to the question "Who benefited most from fraudulent voting activity?" (Continuous scale 0 [Trump / Biden] - 100 [Biden / Trump], the labels' order was counterbalanced across participants). The value of $c$ was set as the reported probability of fraud (if present) benefiting their preferred candidate, divided by 100 for a range of $[0,1]$.

The correlation between participants' fraud beliefs during the original survey and their beliefs during the follow-up survey was computed with a Pearson correlation between the posterior fraud belief after seeing a "Biden wins" map and the likelihood of fraud in favor of Biden (on an ordinal scale) as reported during the follow-up survey, only for participants who were randomly assigned to the "Biden wins" scenario during the original survey. The correlations between the preference strength and $v$, and between the preference strength and $c$, were computed with Pearson correlation.

We derived the predicted posteriors and magnitude of updating for both $F$ and $V$ based on the empirically measured priors and model predictions for participants who successfully completed the follow-up survey, preferred either Trump or Biden (i.e., not "Other") and did not change their preference between the two surveys. For some participants, we could not compute a posterior value 
(nor the update magnitude) because the model yielded a non-defined value due to the specific combination of prior beliefs and hypothetical outcome leading to 0 in the denominator (e.g., viewing a win by the preferred candidate after having a $100 \%$ belief that the opposition would reverse the election through fraud). This happened for 99 participants (out of the 828 participants with complete survey data) when computing the posteriors for $F$ and $V$ for each participant's randomly assigned scenario (hypothetical map), and for 147 total participants when computing the posteriors for $F$ and $V$ for the loss scenario irrespective of the scenario the participant was randomly assigned to. To assess the model fit, we calculated the Pearson correlation between the empirical and predicted values of fraud belief updates and also the root mean squared error (RMSE).

To estimate the proportion of participants who are considered "election-proof", we calculated for each participant the predicted update of $v$ and $f$ for the loss scenario. As described above, we could not derive predicted posterior values for 147 participants. We then computed how much of the overall update (update of $f+$ update of $v$ ) was attributed to each of these two beliefs. For 66 participants, the model predicted no update of both $f$ and $v$ following a loss by their preferred candidate. We report the proportion of the total update attributed to fraud rather than to the true vote belief for the remaining 615 participants. Many of the participants for whom no update of the true winner belief was predicted had $v$ equal to 1 . Since previous literature and the model prediction for posterior belief in fraud suggested that priors of 1 may not reflect a truly certain belief, we further re-estimated the values when $f, v$ and $c$ where restricted to be between 0.05 - 0.95 (i.e., values smaller than 0.05 were converted to 0.05 and values larger than 0.95 were converted to 0.95). This also allowed us to predict the posteriors for all 828 participants.

\section{Acknowledgments}

We thank Professors John M. Carey, Russell Muirhead and Brendan J. Nyhan for providing helpful comments on a previous version of this manuscript. Rotem Botvinik-Nezer is an Awardee of the Weizmann Institute of Science - Israel National Postdoctoral Award Program for Advancing Women in Science.

Funding

Nothing to report.

\section{Author contributions:}

Study design: R.B.-N. and T.D.W.

Data collection: R.B.-N.

Data analysis: R.B.-N.

Supervision, expertise, and feedback: M.J. and T.D.W.

Bayesian model formulation: M.J.

Conceptual framework development: R.B.-N., M.J. and T.D.W.

Writing: R.B.-N., M.J. and T.D.W. 


\section{Competing interests}

Authors declare that they have no competing interests.

Data and materials availability

All data, code, and materials are publicly available at https://doi.org/10.5281/zenodo.5730631 (release v1.0.0).

\section{References}

1. R. S. Nickerson, Confirmation Bias: A Ubiquitous Phenomenon in Many Guises. Rev. Gen. Psychol. 2, 175-220 (1998).

2. M. Jepma, L. Koban, J. van Doorn, M. Jones, T. D. Wager, Behavioural and neural evidence for self-reinforcing expectancy effects on pain. Nature Human Behaviour. 2, 838855 (2018).

3. Z. Kunda, The case for motivated reasoning. Psychol. Bull. 108, 480-498 (1990).

4. T. Sharot, N. Garrett, Forming Beliefs: Why Valence Matters. Trends Cogn. Sci. 20, 25-33 (2016).

5. B. M. Tappin, L. van der Leer, R. T. McKay, The heart trumps the head: Desirability bias in political belief revision. J. Exp. Psychol. Gen. 146, 1143-1149 (2017).

6. H. M. Dorfman, R. Bhui, B. L. Hughes, S. J. Gershman, Causal Inference About Good and Bad Outcomes. Psychological Science. 30 (2019), pp. 516-525.

7. S. J. Gershman, How to never be wrong. Psychon. Bull. Rev. 26, 13-28 (2019).

8. A. Jern, K.-M. K. Chang, C. Kemp, Belief polarization is not always irrational. Psychol. Rev. 121, 206-224 (2014).

9. U. Hahn, A. J. L. Harris, What Does It Mean to be Biased. Psychology of Learning and Motivation (2014), pp. 41-102.

10. M. Kim, B. Park, L. Young, The Psychology of Motivated versus Rational Impression Updating. Trends Cogn. Sci. 24, 101-111 (2020).

11. S. Iyengar, K. S. Hahn, Red Media, Blue Media: Evidence of Ideological Selectivity in Media Use. J. Commun. 59, 19-39 (2009).

12. E. Bakshy, S. Messing, L. A. Adamic, Political science. Exposure to ideologically diverse news and opinion on Facebook. Science. 348, 1130-1132 (2015).

13. J. J. Van Bavel, S. Rathje, E. Harris, C. Robertson, A. Sternisko, How social media shapes polarization. Trends Cogn. Sci. 25, 913-916 (2021). 
14. B. F. Schaffner, S. Luks, Misinformation or expressive responding? What an inauguration crowd can tell us about the source of political misinformation in surveys. Public Opin. $Q$. 82, 135-147 (2018).

15. A. J. Berinsky, Telling the Truth about Believing the Lies? Evidence for the Limited Prevalence of Expressive Survey Responding. J. Polit. 80, 211-224 (2018).

16. N. Bermeo, On Democratic Backsliding. Journal of Democracy. 27, 5-19 (2016).

17. K. Clayton, N. T. Davis, B. Nyhan, E. Porter, T. J. Ryan, T. J. Wood, Elite rhetoric can undermine democratic norms. Proc. Natl. Acad. Sci. U. S. A. 118 (2021), doi:10.1073/pnas.2024125118.

18. S. Levitsky, D. Ziblatt, How Democracies Die (Crown, 2018).

19. N. Berlinski, M. Doyle, A. M. Guess, G. Levy, B. Lyons, J. M. Montgomery, B. Nyhan, J. Reifler, The Effects of Unsubstantiated Claims of Voter Fraud on Confidence in Elections. Journal of Experimental Political Science, 1-16 (2021).

20. M. W. Sances, C. Stewart, Partisanship and confidence in the vote count: Evidence from U.S. national elections since 2000. Elect. Stud. 40, 176-188 (2015).

21. C. J. Anderson, A. Blais, S. Bowler, T. Donovan, O. Listhaug, in Losers' Consent (Oxford University Press, 2005).

22. A. M. Enders, J. E. Uscinski, C. A. Klofstad, K. Premaratne, M. I. Seelig, S. Wuchty, M. N. Murthi, J. R. Funchion, The 2020 presidential election and beliefs about fraud: Continuity or change? Elect. Stud. 72, 102366 (2021).

23. S. Flaxman, S. Goel, J. M. Rao, Filter Bubbles, Echo Chambers, and Online News Consumption. Public Opin. Q. 80, 298-320 (2016).

24. T. Sharot, C. R. Sunstein, How people decide what they want to know. Nat Hum Behav. 4, $14-19(2020)$.

25. C. J. Charpentier, E. S. Bromberg-Martin, T. Sharot, Valuation of knowledge and ignorance in mesolimbic reward circuitry. Proc. Natl. Acad. Sci. U. S. A. 115, E7255-E7264 (2018).

26. M. Vlasceanu, A. Coman, The Impact of Social Norms on Belief Update (2020) (available at https://psyarxiv.com/gsem6/download?format=pdf).

27. A. Pereira, E. Harris, J. J. Van Bavel, Identity concerns drive belief: The impact of partisan identity on the belief and dissemination of true and false news. Group Process. Intergroup Relat., 13684302211030004 (2021).

28. J. S. Beck, Cognitive Behavior Therapy, Third Edition: Basics and Beyond (Guilford Publications, 2020).

29. N. F. Johnson, N. Velásquez, N. J. Restrepo, R. Leahy, N. Gabriel, S. El Oud, M. Zheng, P. 
Manrique, S. Wuchty, Y. Lupu, The online competition between pro- and anti-vaccination views. Nature. 582, 230-233 (2020).

30. J. N. Druckman, M. C. McGrath, The evidence for motivated reasoning in climate change preference formation. Nat. Clim. Chang. 9, 111-119 (2019).

31. R. Bertoldo, C. Mays, G. Böhm, W. Poortinga, M. Poumadère, E. Tvinnereim, A. Arnold, K. Steentjes, N. Pidgeon, Scientific truth or debate: On the link between perceived scientific consensus and belief in anthropogenic climate change. Public Underst. Sci. 28, 778-796 (2019).

32. L. Litman, J. Robinson, T. Abberbock, TurkPrime.com: A versatile crowdsourcing data acquisition platform for the behavioral sciences. Behav. Res. Methods. 49, 433-442 (2017). 


\title{
Supplementary Materials
}

\author{
Fraud Beliefs Following the 2020 U.S. Presidential Election: \\ A Belief Systems Analysis \\ Rotem Botvinik-Nezer ${ }^{1}$, Matt Jones ${ }^{2}$, Tor D. Wager ${ }^{1 *}$ \\ Correspondence to: Tor.D.Wager@dartmouth.edu
}

\section{Extended results}

Biased prior beliefs: win beliefs, fraud beliefs and the relationship between them

The predictions described in this section are based mainly on the desirability bias hypothesis, as they were pre-registered prior to the development of the Bayesian model. Based on our hypothesis that fraud beliefs are used as an alternative causal explanation that absorbs blame for undesired results, we predicted that the more participants believed their candidate was losing, the more they would believe in fraud prior to seeing the hypothetical map (i.e., a negative main effect of prior win belief on prior fraud belief). We further predicted that this effect would be stronger for participants with stronger preference (i.e., an interaction effect between prior win belief and preference strength on prior fraud belief), since their motivation to believe their candidate is winning is higher. Finally, we suspected an additional countervailing positive relationship between prior win belief and prior fraud belief among Republicans, due to a presumed correlation in exposure to claims that their candidate is winning and that the election is substantially influenced by fraud (19). Therefore, the aforementioned negative effect of prior win belief was predicted mainly for Democrats.

Following our pre-registered analysis plan, we tested our predictions with multiple regression, predicting prior fraud belief based on the prior win belief, the preferred candidate (Biden / Trump; a factor with contrasts coded as 1 for Biden and -1 for Trump), the preference strength, the interaction between the preferred candidate and preference strength, and the interaction between prior win belief and preference strength (the first main effect and the last interaction effect were our effects of interest). Participants' age and order of survey submission were included in the model as covariates. The participant's state of residence was not included as it did not significantly reduce residual variance while substantially increasing model complexity. The same model was also tested separately for each partisan subgroup (preferred candidate $=$ Democrat and preferred candidate $=$ Republican), where the preferred candidate main effect and interaction were omitted. All numeric variables in the model (dependent and independent) were z-scored.

Prior win belief was not negatively related to prior fraud belief across the sample (multiple regression, $\beta=0.01, p=.728$; Table $\mathrm{S} 5$ ). As suspected, there was a difference between the partisan groups (Fig. S2D): While the relationship between prior win belief and prior fraud belief was negative for Democrats $(\beta=-0.12, p<.001$; Table S6), it was positive for Republicans $(\beta=0.10$, $p=.004$; Table S7). Contrary to our prediction, the relationship between the prior win belief and the prior fraud belief did not significantly correlate with the preference strength, neither across the sample $(p=.357)$ nor for Democrats only $(p=.063)$ or Republicans only $(p=.484)$.

For completeness (and although it was not included in our pre-registration), we further report here the results of the full multiple regression model with all interaction terms (prior fraud 
belief as the dependent variable, the prior win belief, the preference strength and the preferred candidate as the independent variables with all main effects and interaction terms, and age and order of survey submission as covariates; all numerical variables were z-scored). Similar to the results reported above, the prior win belief was not significantly related to the prior fraud belief when both partisan groups were combined (multiple regression, $\beta=-0.01, p=.552$ ), and the interaction between prior win belief and preference strength was also not significant $(p=.507)$. The interaction between the preferred candidate and the prior win belief was significant $(p<.001)$, in line with the partisan results reported above. The main effect of preferred candidate and the interaction between the preferred candidate and the preference strength were also significant (both $p s<.001)$, in line with the partisan difference in prior fraud belief reported in the main text (Republicans had significantly stronger prior fraud beliefs than Democrats, and this difference was larger for participants with stronger preferences).

Overall, these results are more consistent with biased prior beliefs leading to biased posterior beliefs than with biased inference. Only Democrats believed in fraud more when they thought they are more likely to lose, and this effect is unlikely to result from motivated reasoning since it did not scale with preference. Republicans, on the other hand, believed in fraud more the more they believed they were going to win. These prior beliefs are consistent with messages communicated by leaders from each party.

\section{Partisan differences in belief updating}

Effects on belief update across the entire sample are reported in the main text. To test whether there are partisan differences in the update of fraud belief, we tested the linear models described in the main text, for the win and loss scenarios, separately for each partisan group. When presented with a map showing a Republican win (Table S8), Democrats' fraud belief significantly increased $\left(M=20.47, S D=33.42, t_{(515)}=13.92, p<.001\right)$. This increase was larger when the prior win belief was higher and therefore the loss was more surprising $(\beta=0.23, p=.001)$, but not when their preferences were stronger $(\beta=0.03, p=.570)$. When Democrats were presented with a winning map (see Table S9), their fraud belief significantly decreased $\left(M=-7.03, S D=18.32, t_{(515)}=-8.71\right.$, $p<.001)$. This update was larger when their preference for Biden was stronger $(\beta=-0.07, p<$ $.001)$; however, there was no effect of the prior win belief $(\beta=-0.02, p=.545)$, which means that fraud beliefs were not related to how surprising the outcome was in this condition.

Republicans, on the other hand, did not significantly increase fraud belief following a losing map $\left(M=0.4, S D=21.61, t_{(307)}=0.32, p=.746\right)$, likely because many republicans reported very high prior fraud belief, and therefore could not increase it much in the loss scenario. However, they did increase their fraud belief significantly more when their preference was stronger $(\beta=0.18, p<$ .001 ; Table S10) and when the loss was more surprising $(\beta=0.09, p=.005)$. Following a winning map (see Table S11), Republicans' fraud belief significantly decreased $(M=-34.98, S D=35.77$, $\left.t_{(301)}=-16.99, p<.001\right)$, and decreased significantly more when their preference for Trump was stronger $(\beta=-0.15, p=.008)$ but not when they were more surprised by the result $(\beta=-0.02, p=$ $.714)$.

In summary, participants believed in fraud less when a win was observed and more when a loss was observed (besides one discrepancy which may have been affected by maximal prior beliefs for some participants). When losing, but not when winning, the update in fraud was larger when the result was more surprising. In all conditions except for Democrats following a loss, the update 
was larger when the preference was stronger (i.e., when the map was more desired in the win scenario or more undesired in the loss scenario).

\section{Update of fraud belief: robustness across model variants and covariates}

As reported in the main text, we found that participants' fraud belief update was significantly related to the scenario (i.e., whether their preferred candidate won or lost), the strength of their preference and the prior win belief. Here, we report the results with some model variations. First, the regression models reported in the main text controlled for the prior fraud belief, the preferred candidate, the participant's age, and the order of survey submission during data collection. The inclusion of the participants' age as a covariate led to the exclusion of participants who chose not to report their age: 17 participants in the loss scenario and 12 participants in the win scenario. We verified that the inclusion of these participants does not change the results by testing the same models without the age covariates, and indeed observed similar results to those reported in the main text. We also verified that our results are not solely driven by the inclusion of the specific covariates in our models. When testing the same models without the covariates, we observed the same pattern of results, except that the magnitude of decreases in fraud belief in the win scenario was also significantly related to the prior win belief, such that updates were smallest when the outcome was expected.

Second, as can be seen in Fig. 1D, the distribution of the preference strength was positively skewed. To verify our multiple linear regression reported in the main text for the fraud belief update, we split the preference strength into three categories: Low-moderate (preference strength of $0-80, N=401)$, high $(81-99, N=375)$ and maximal $(100, N=866)$. These categories were chosen to maximize the balance across categories, given the limitation that the highest category necessarily includes most of the sample. We conducted a one-way ANCOVA with fraud belief update as the dependent variable, the categorical preference strength as the independent variable, and the prior fraud belief, prior subjective probability of a win by the preferred candidate, preferred candidate, age, and order of survey submission as covariates, separately for the loss and the win scenarios (similar to the variables included in the multiple linear regression reported in the main text). In both scenarios, we found a significant main effect for the preference strength (loss scenario: $\left.F_{(2,799)}=5.63, p=.004\right)$; win scenario: $\left.F_{(2,798)}=14.59, p<.001\right)$. We then tested the simple effects with the Tukey post-hoc test. For the loss scenario, participants from the highest preference strength category increased their fraud belief significantly more than participants from the lowest preference strength category (adjusted $p<.001$ ), but the difference between the middle category and any of the extreme ones was not significant. For the win scenario, participants from the highest preference strength category decreased their fraud belief significantly more than participants in the middle preference strength category (adjusted $p<.001$ ) and in the lowest preference strength category (adjusted $p<.001$ ), but the difference between the middle category and the lowest one was not significant.

\section{Update of fraud belief: pre-registered analysis}

We planned to model the update of fraud belief with a multiple linear regression including the following predictors: Map type (Republican vs. Democrat win, a factor with contrasts set as 1 for a Democrat win and -1 for a Republican win), preference strength towards the Republican candidate (preference strength was multiplied by -1 for Democrat participants), prior subjective 
probability of a Republican win (the prior win belief for Republican participants and 100 minus the prior win belief for Democrat participants), the interaction between map type and preference strength towards the Republican candidate, and the interaction between map type and prior belief about a Republican win. The two interactions were our effects of interest, along with specific simple effects of preference strength and prior win belief on the update of fraud belief in each map condition. To simplify the model and interpretation, focus on main rather than interaction effects, and examine both the direction of the update and its magnitude, we instead decided to test the preference strength and the prior win belief separately for the loss and win scenarios. We further tested these models separately for each partisan group.

While the results of the revised models are described in the main text, here we describe the (similar) results of the pre-registered model for full transparency. All numerical variables were z-scored, and the age and order of survey submission were included as covariates (but their inclusion does not change the results of interest in a meaningful way).

As predicted, both interactions were significant (both ps $<.001$ ), such that fraud beliefs increased when the outcome was unexpected or undesired. Simple effects further followed our predictions: When the map showed a Democrat win, both the preference towards the Republican candidate (linear regression, $\beta=0.12, p<.001$ ) and the prior belief in a Republican win (linear regression, $\beta=0.09, p<.001$ ) had a significant positive effect on the update of fraud belief. When the map showed a Republican win, both the preference towards the Republican candidate (linear regression, $\beta=-0.81, p<.001$ ) and the prior belief in the Republican candidate's win (linear regression, $\beta=-059, p<.001$ ) had a significant negative effect on the update of fraud belief. In addition, the update of fraud belief was significantly positive following a losing map (one sample t-test, $\left.M=12.97, t_{(823)}=11.97, p<.001\right)$. These effects are consistent with the main empirical findings reported in the main text, demonstrating that the update of fraud belief was significantly affected by both the desirability of the outcome and how surprising it was.

When exploring the update of fraud belief separately for each map and partisan group, we found that the update was larger (in absolute value) following a Republican-win compared to a Democratic-win map. This was likely driven by floor and ceiling effects: While many Republicans were very confident that fraud was affecting the election outcome before seeing the map and therefore could not increase their belief much more following a Democratic-win map, many Democrats did not believe fraud was affecting the election outcome before seeing the map and therefore could not decrease their belief much further following a Democratic-win map (Fig. S3A).

\section{Follow-up survey: divergent beliefs}

The follow-up survey revealed that participants strongly believed that their preferred candidate would have won based on the true votes, and that if fraud was committed, it benefited the opposite candidate. Participants were asked who would have won in a fraudless election (i.e., one where every vote reflects the true will of the American people). We found that the belief about the fraudless winner significantly depended on the preferred candidate at the time of the follow-up survey (Chi-squared test, $\chi_{(2)}^{2}=583.3, p<.001$; participants who indicated "Other" as their preferred candidate were excluded from this analysis since their inclusion resulted in cells with frequencies that were too low for reliable chi-squared approximation; Fig. S7A). While $69.49 \%$ of the Republican participants claimed Trump would have won in the absence of fraud $(N=272$, $13.24 \%$ said they did not know and $17.28 \%$ believed Biden would have won), $94.93 \%$ of the 
Democratic participants claimed that Biden would have won $(N=612,4.08 \%$ said they did not know who would have won, and $0.98 \%$ indicated Trump would have).

When asked who benefited most from fraudulent activity, participants strongly believed it was their dispreferred candidate: $94.49 \%$ of the Republicans believed fraudulent activity favored Biden and only $1.84 \%$ believed it favored Trump $(3.68 \%$ believed there was no fraud or it benefited both equally). In contrast, $53.92 \%$ of the Democrats believed fraud favored Trump and only 13.56\% believed it favored Biden (32.52\% believed there was no fraud or it benefited both equally; Fig. S7B). On a scale of 0-100, with 0 indicating fraud was $100 \%$ in favor of Biden, 100 indicating it was $100 \%$ in favor of Trump, and 50 indicating there was no fraud or it benefitted both equally, Democrats were significantly higher than $50\left(M=63.59, S D=24.16, t_{(611)}=13.91, p<.001\right)$ and Republicans were significantly lower than $50\left(M=10.08, S D=18.37, t_{(271)}=-35.83, p<.001\right)$. Moreover, in both partisan groups, a vast majority of participants believed that fraud in favor of their preferred candidate was unlikely or extremely unlikely to significantly affect the election outcome: $87.13 \%$ of the Republican participants and $94.44 \%$ of the Democratic participants who completed the follow-up survey (Fig. S7C-D). Ordinal logistic regression (Table S12) indeed indicated a significant interaction between the preference and the belief about the fraud beneficiary $(p<.001)$. Republicans and Democrats also diverged with regard to the activities they considered as fraud and the candidate they believed benefited most from each such activity (Fig. S8). 
A
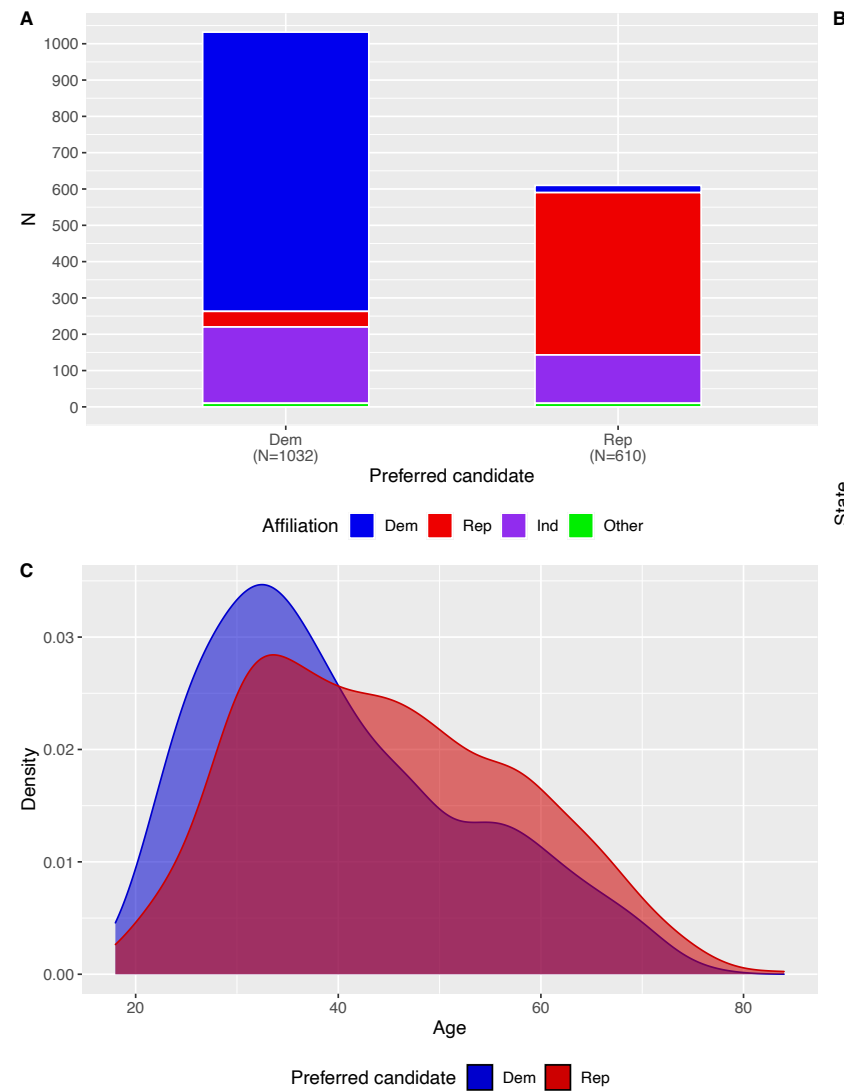

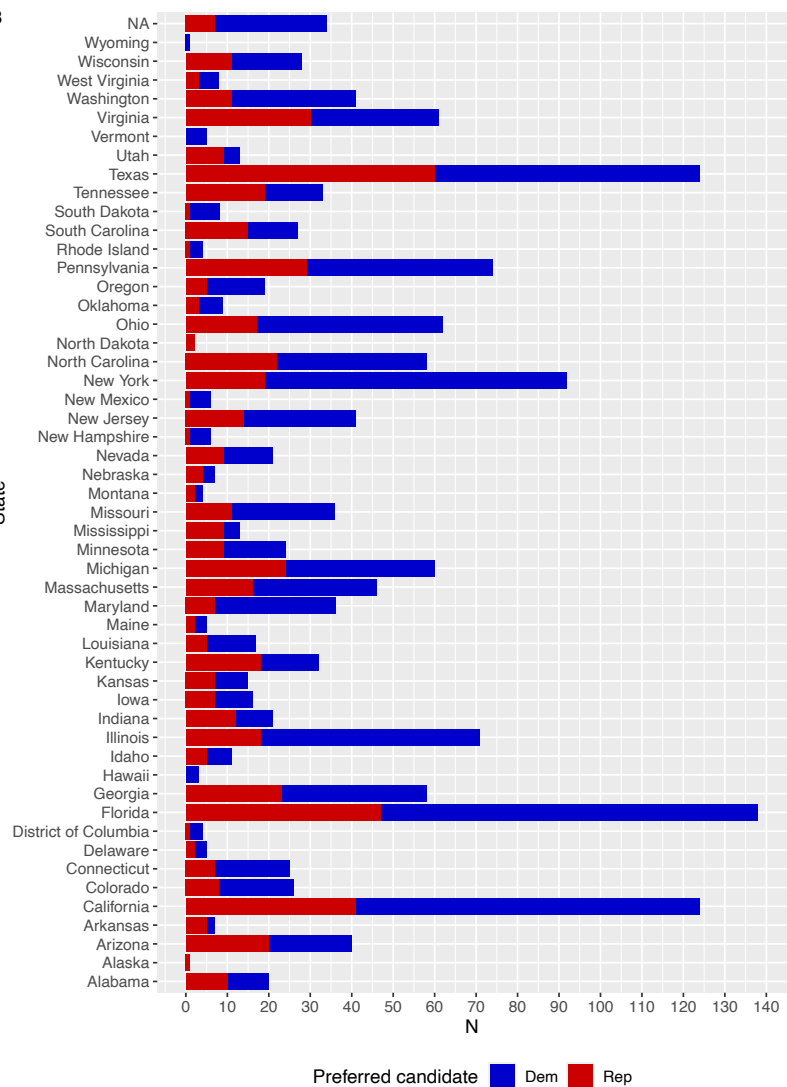

Fig. S1. Demographic and partisan affiliation of participants.

(A) Number of participants for each combination of preferred candidate (x axis) and political affiliation (color). (B) Number of participants from each state who preferred each candidate (color). (C) Kernel density plot of participants' age as a function of the preferred candidate (color). Preferred candidate: Dem = Biden; Rep = Trump; Affiliation: Dem = Democrat; Rep = Republican; Ind = Independent; Other. 

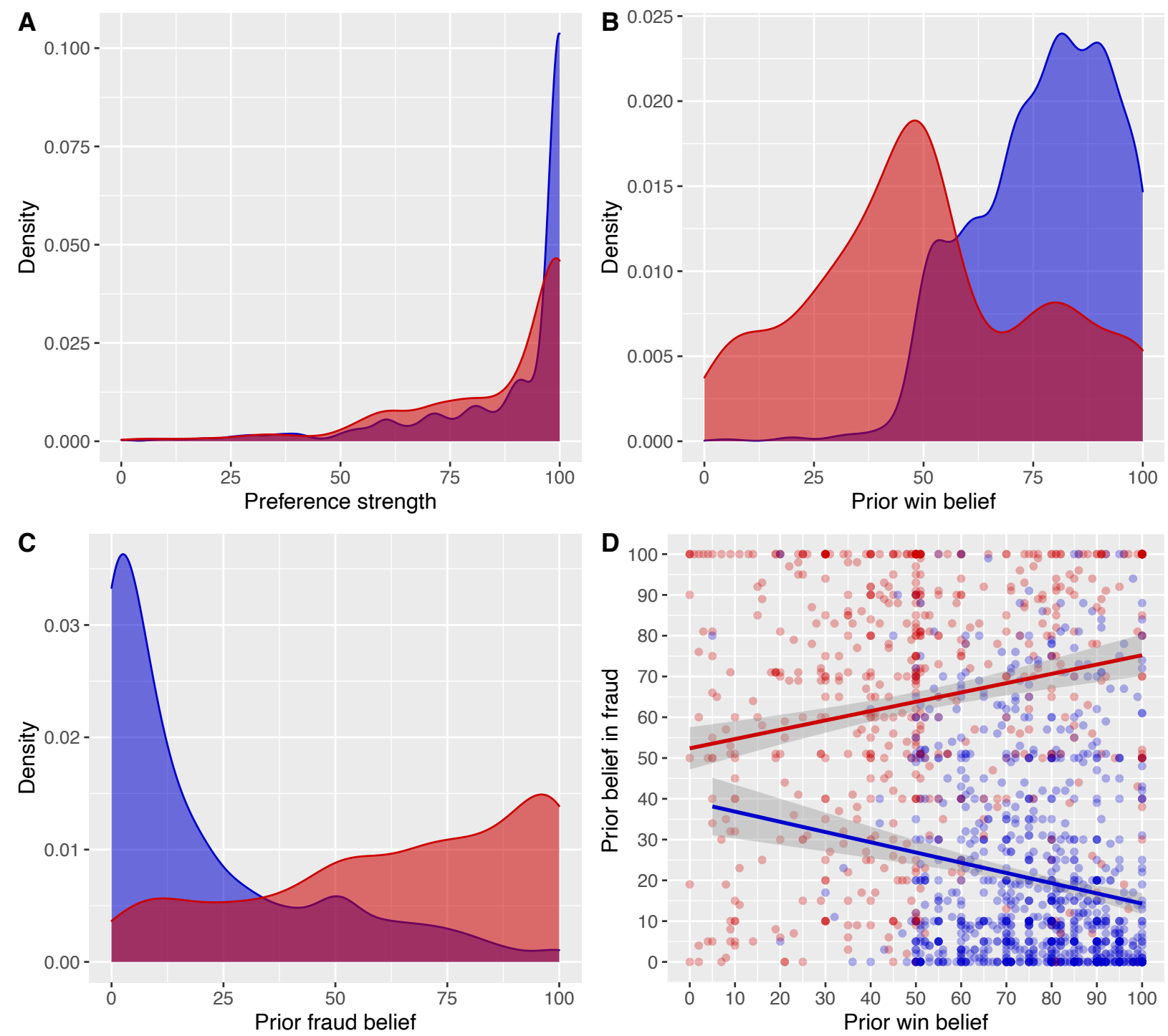

Preferred candidate

Dem $\square$ Rep

\section{Fig. S2. Preference and prior belief data.}

Kernel density plots of: (A) preference strength; (B) prior subjective probability of win by the preferred candidate; and (C) prior fraud belief. (D) Scatterplot for prior fraud belief as a function of prior subjective probability of win by the preferred candidate. Gray area represents $95 \%$ confidence interval; each point represents a single participant (with $30 \%$ opacity). Color represents the preferred candidate: $\mathrm{Rep}=$ Trump (red); Dem = Biden (blue). 


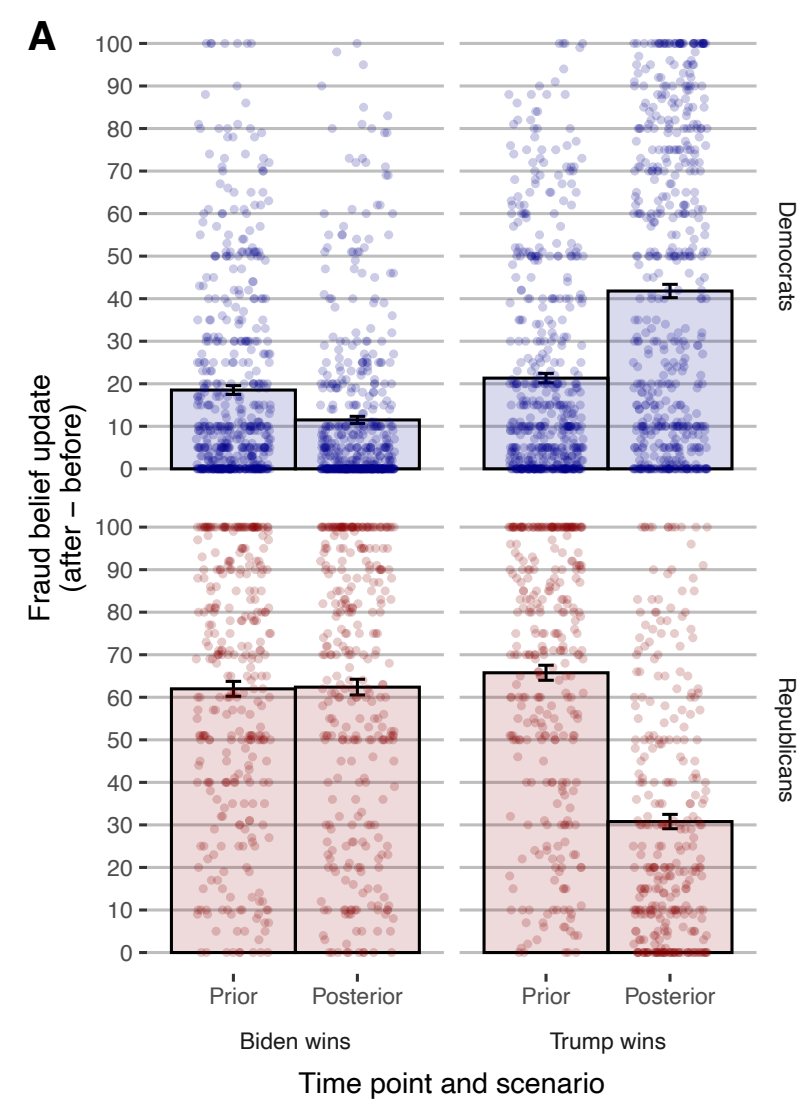

Preferred candidate

Democrats

Republicans
B

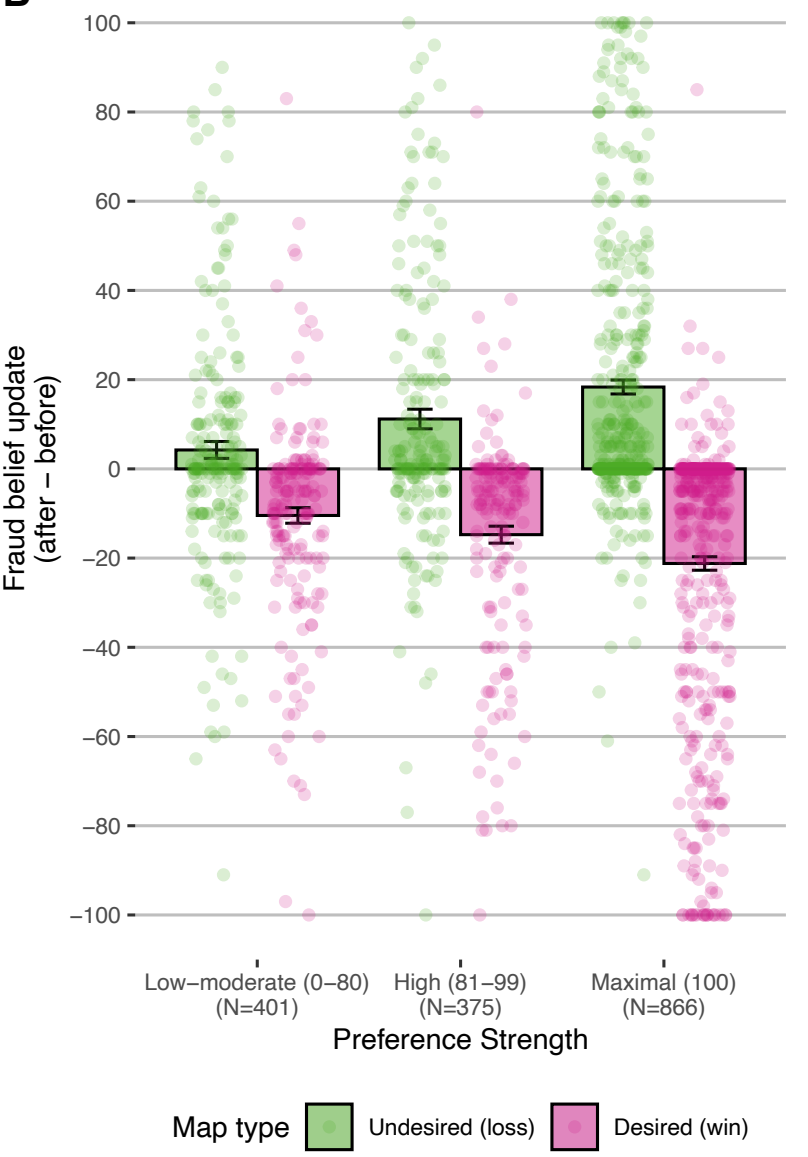

\section{Fig. S3. Desirability effects.}

(A) Prior and posterior (following presentation of the hypothetical maps) fraud beliefs for Democrats and Republicans, for each scenario. (B) Fraud belief update as a function of the scenario (loss or win according to the hypothetical map) and the preference strength (categorized into three ordered categories). Points represent single participants, and error bars represent standard error of the mean across participants. 

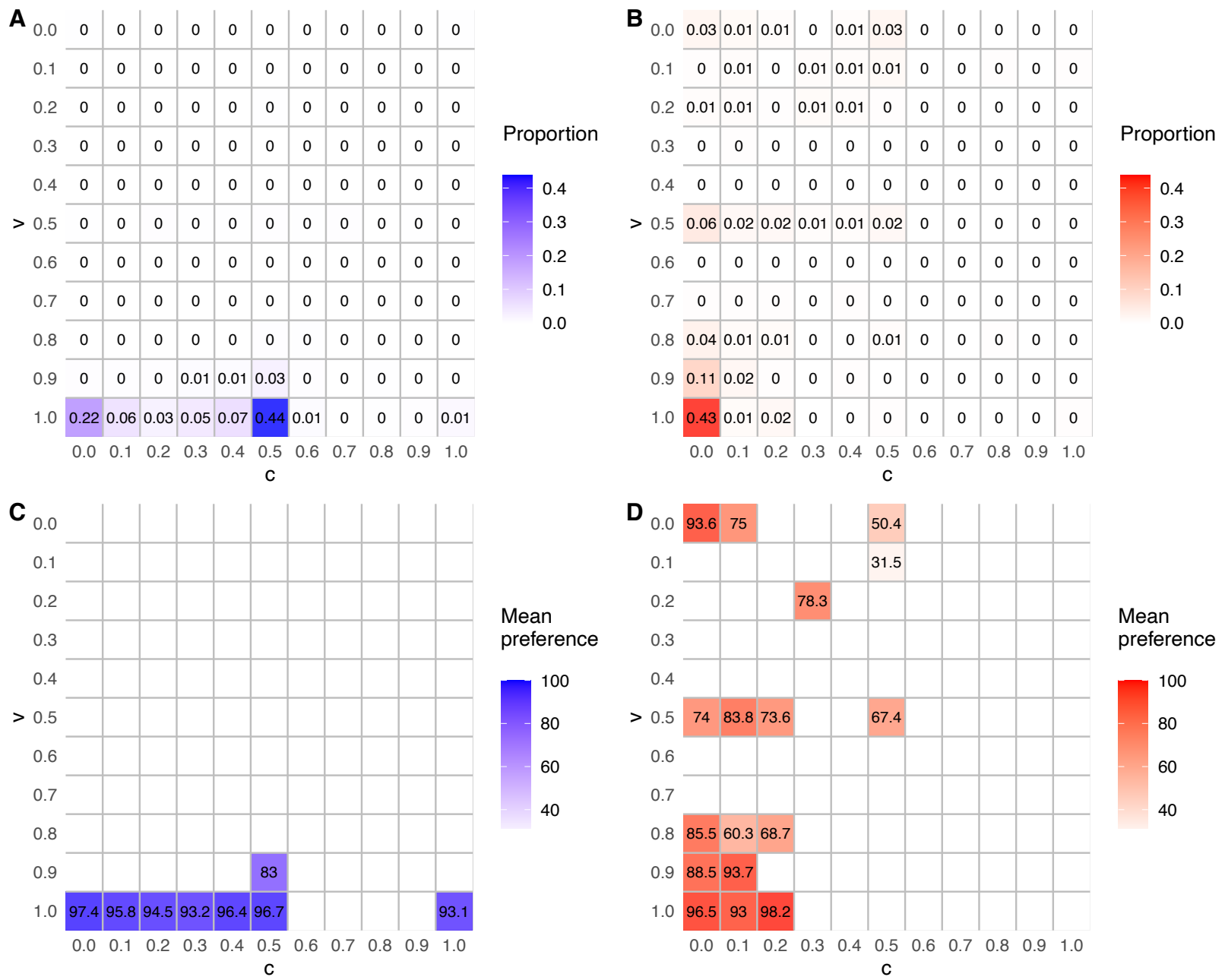

Fig. S4. Empirically measured priors from the follow-up survey.

(A-B) Heat maps for the proportion of participants with each combination of $v$ (probability of the preferred candidate winning the true votes) and $c$ (probability of fraud, if present, favoring the preferred candidate), based on the follow-up sample, for (A) Democratic participants and (B) Republican participants. (C-D) Heat map of the mean preference strength of participants for each combination of $v$ and $c$, for (C) Democratic participants and (D) Republican participants. For panels $\mathrm{C}$ and $\mathrm{D}$, mean preference is shown only for cells with $>1 \%$ of participants. 
A

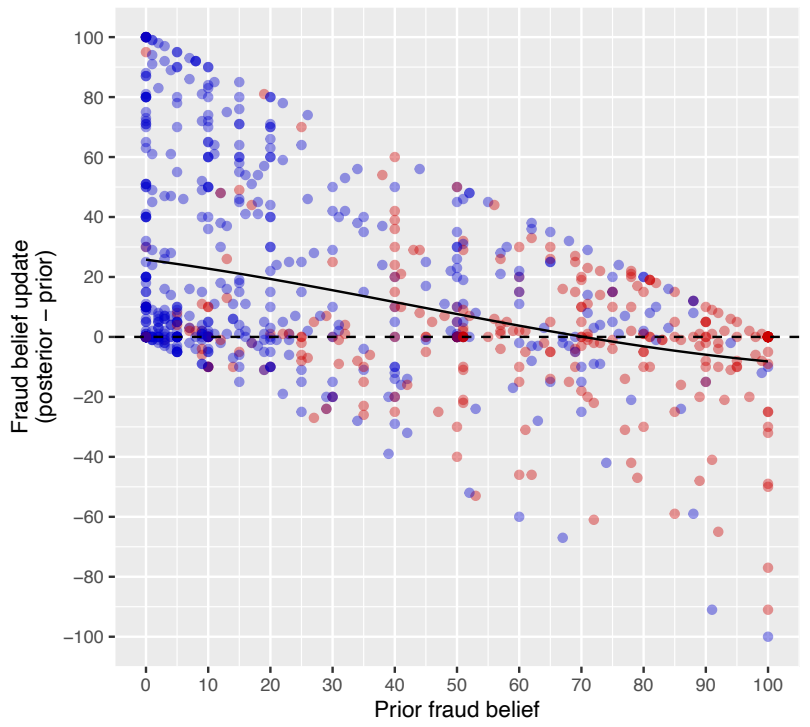

C Loss: Predicted update

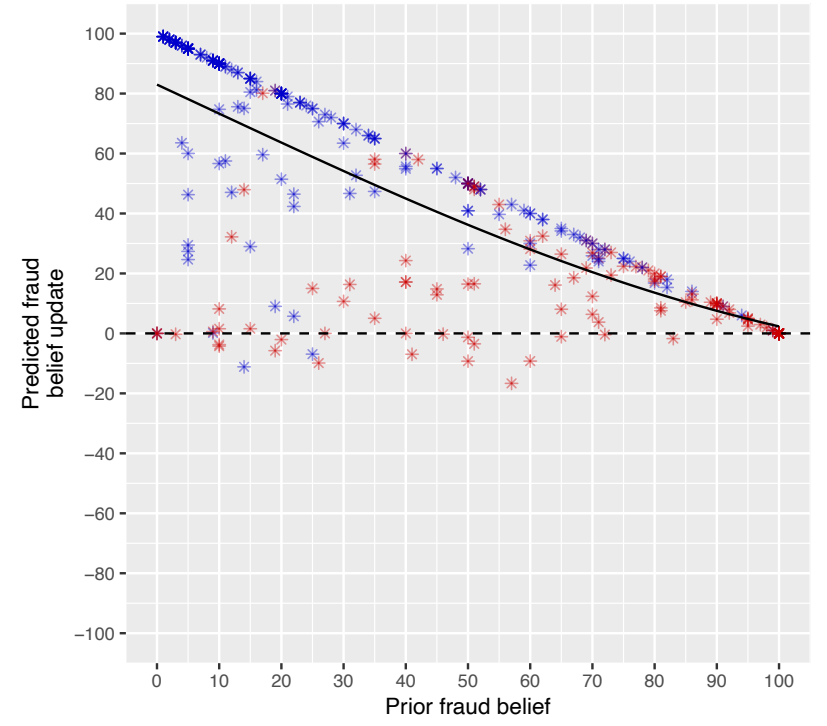

B Win: Empirical update

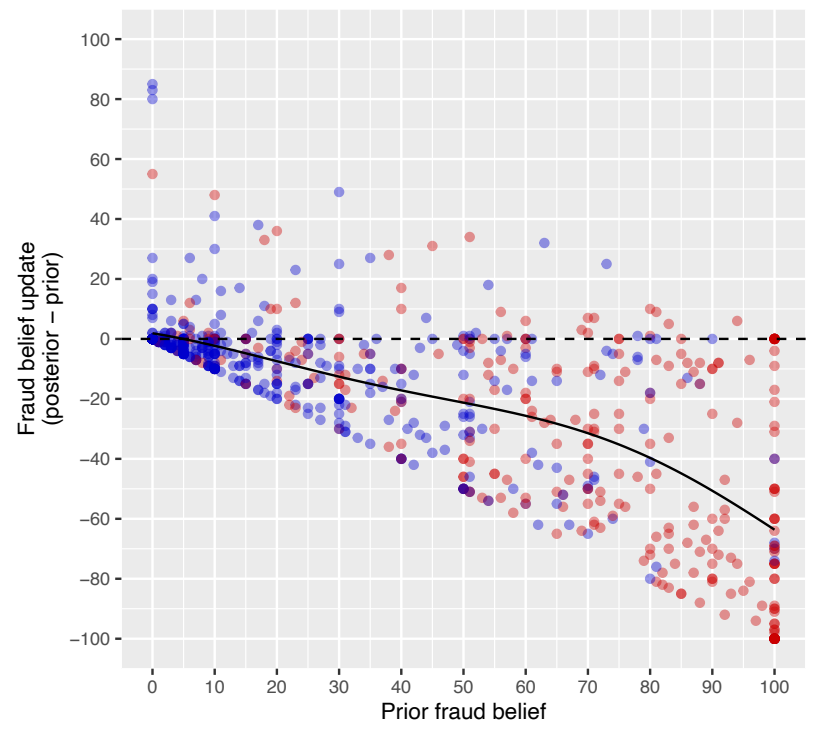

D Win: Predicted update

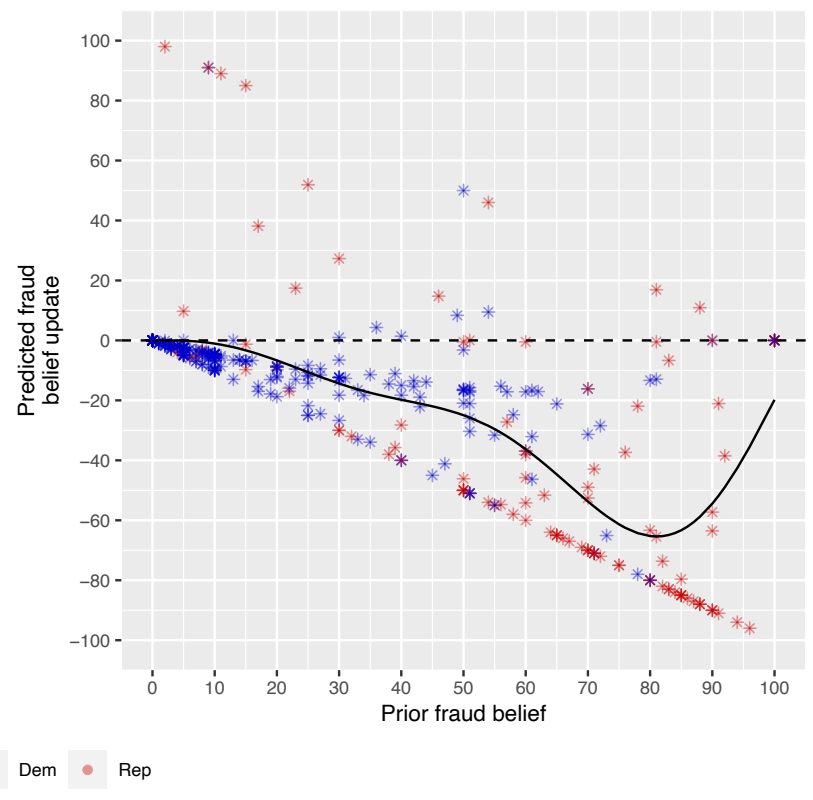

Fig. S5. Empirical data vs. model predictions.

Empirical fraud belief update as a function of prior fraud belief, when the preferred candidate (A) loses or (B) wins. Predicted fraud belief update based on the Bayesian model, when the preferred candidate $(\mathbf{C})$ loses or $(\mathbf{D})$ wins. The solid lines are the means smoothed with Gaussian Process regression. 


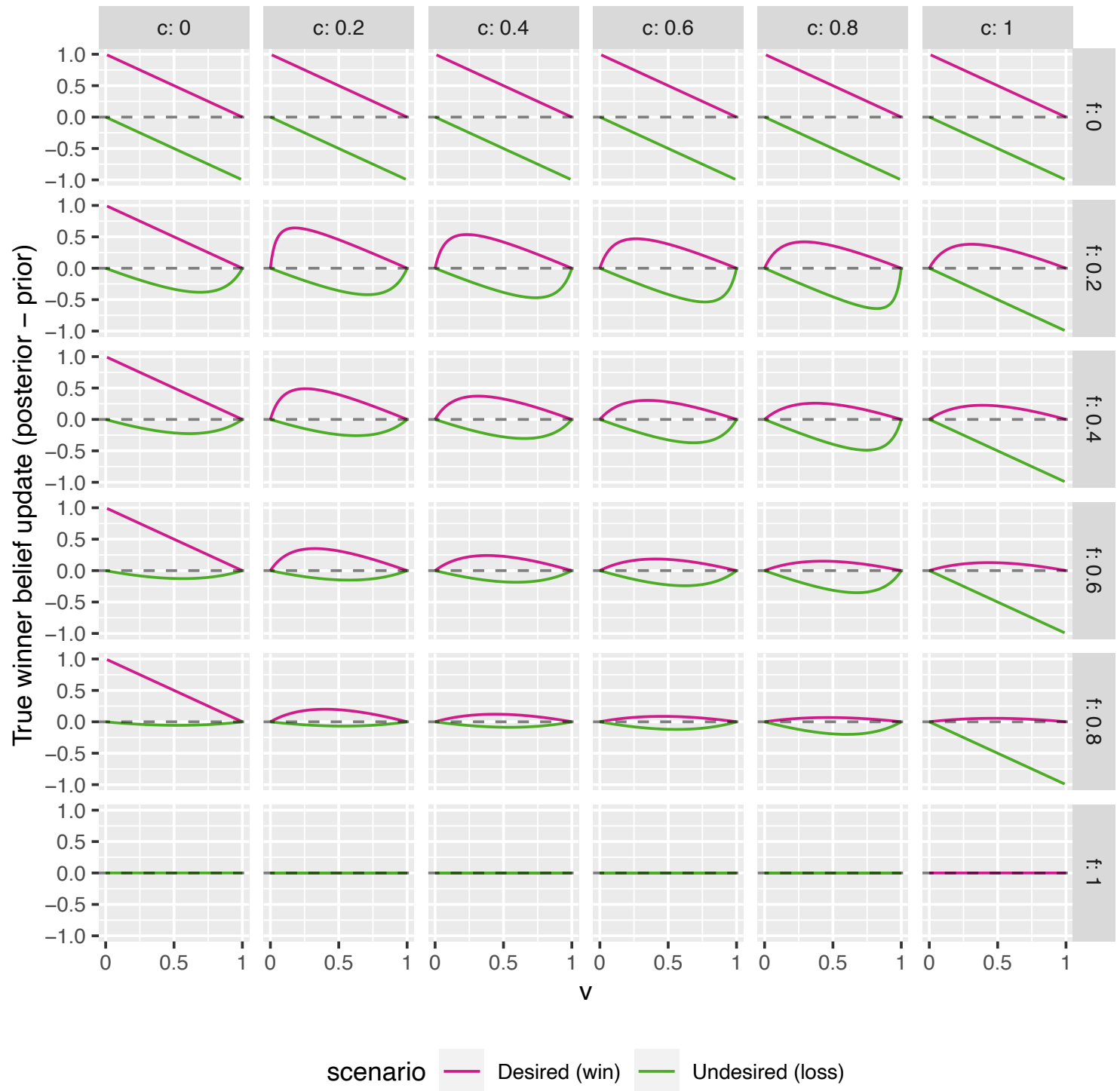

Fig. S6. True winner belief update.

The Bayesian model's predicted update of the belief that the preferred candidate is the true winner $(V)$ over $v, f$ and $c$ parameter space. 

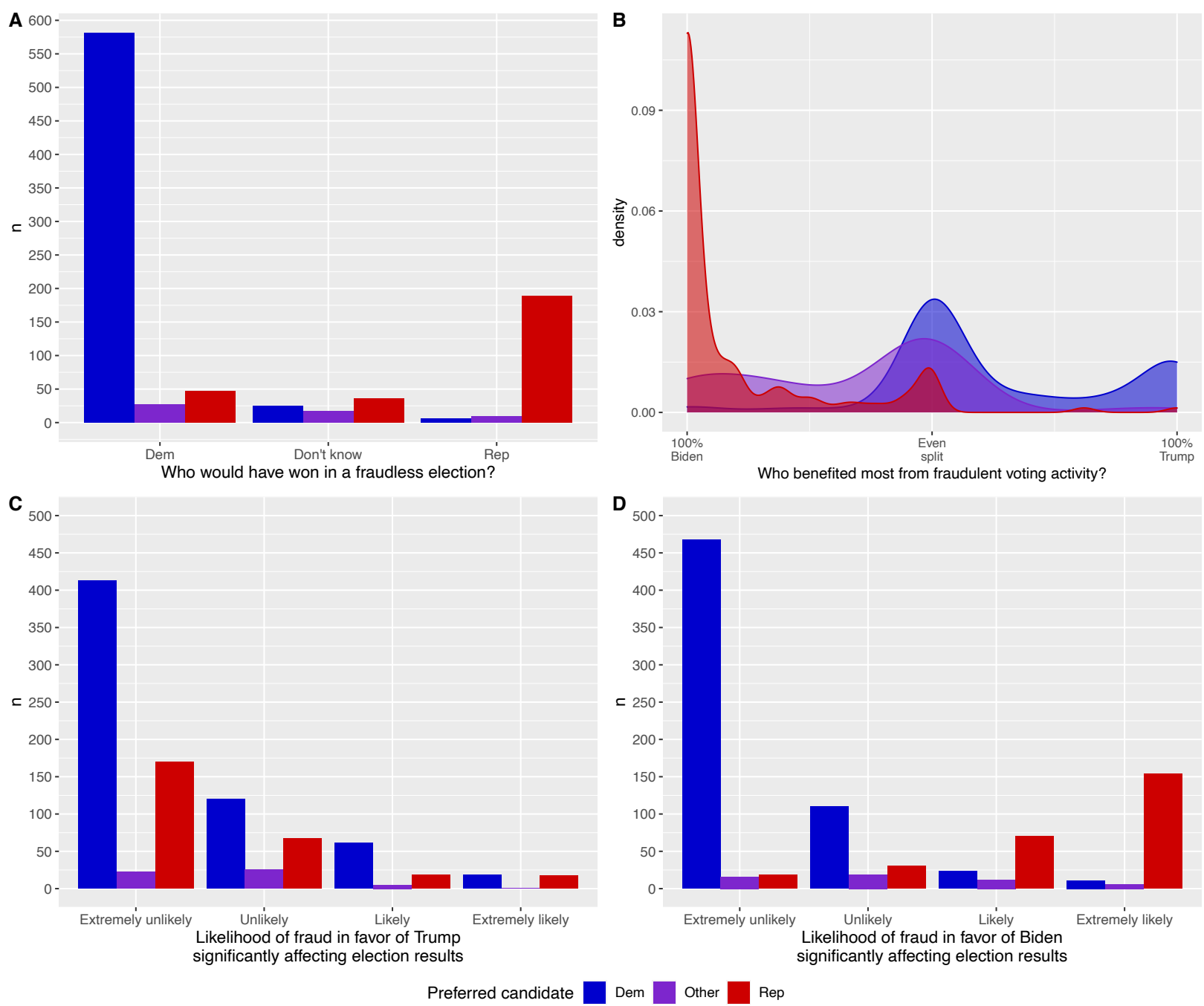

Fig. S7. Follow-up survey: fraud probability and benefiting candidate.

(A) The frequency of beliefs about the winner in a fraudless election. (B) Density plot for the candidate benefiting more from fraudulent activity. (C) The number of participants reporting each level of fraud probability in favor of Trump and (D) in favor of Biden. Color represents the preferred candidate at the time of the follow-up survey. 
A

Social media campaigns spreading false information

Mis-reporting of final vote counts by state electoral officials

Mis-recording of votes by state vote-counters and state electoral officials

Tampering with electronic voting systems

Mail-in ballot fraud

Ballot manipulation or ballot-box stuffing

$\varangle$ Vote buying / voter impersonation / misuse of proxy votes

Electorate manipulation prior to election day

False news stories by news aggregator services (e.g., Reuters, Allied Press) -

False news stories by "left-wing" media

False news stories by "right-wing" media
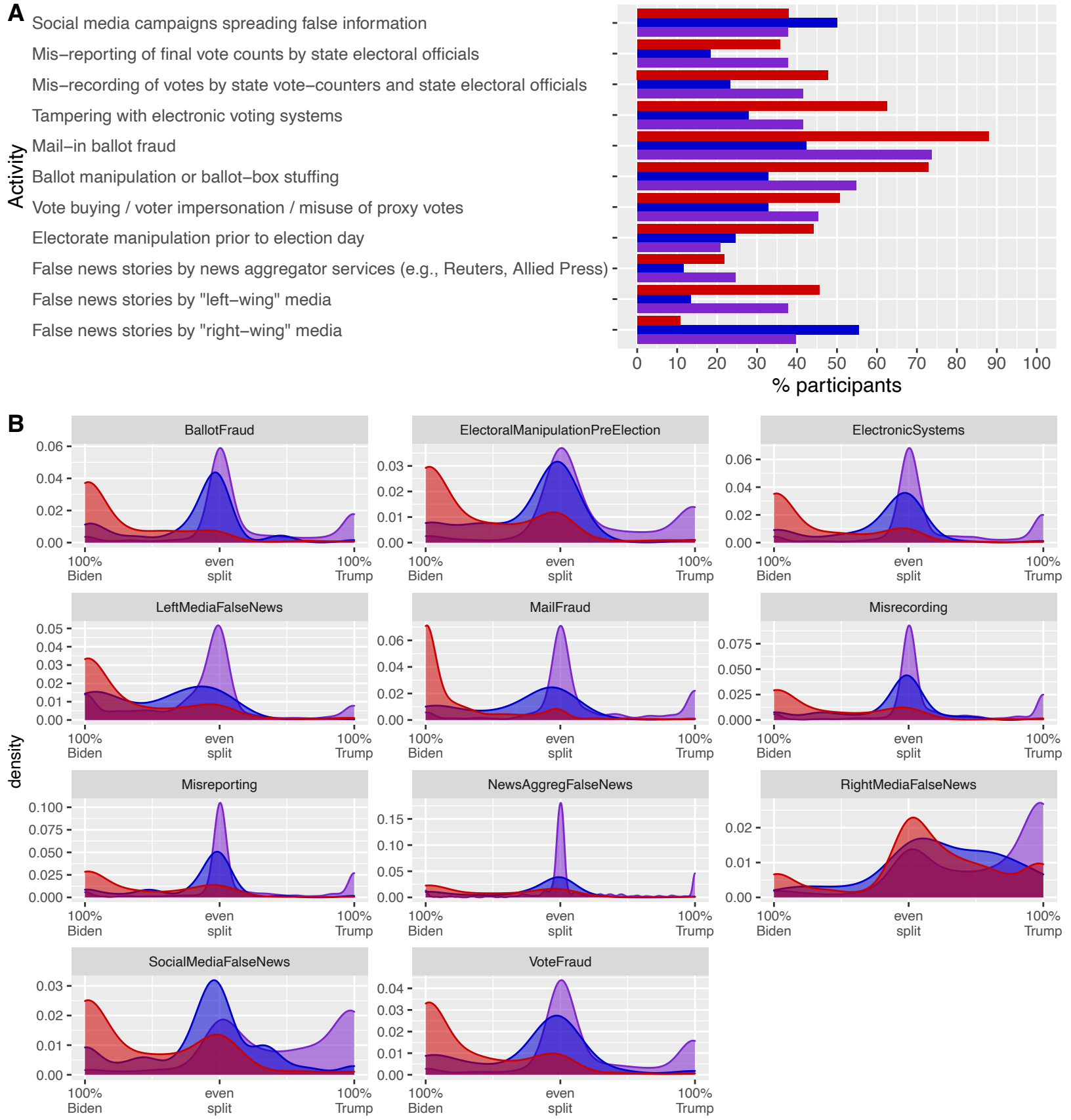

Preferred candidate

Other

Dem

Rep

Fig. S8. Follow-up survey: fraud types.

(A) For each activity, the percentage of participants indicating they considered this specific activity as fraud is presented. (B) Density plots for different types of activities and their believed benefiting candidate. Colors represent the preferred candidate. $N$ s: Other $=53$; Dem $=612$; Rep $=272$ 
Table S1.

\begin{tabular}{|c|c|c|}
\hline Order & Question & Answer structure \\
\hline 1 & Are you a U.S. Citizen or permanent resident? & Yes / No (participants answering "no" were excluded) \\
\hline 2 & What is your political affiliation? & Republican / dDemocrat / Independent / Other (explain) \\
\hline 3 & $\begin{array}{l}\text { Who is your preferred presidential candidate in the current } \\
\text { elections? }\end{array}$ & Donald Trump / Joe Biden / Other \\
\hline 4 & How strong is your preference? & $\begin{array}{l}\text { Continuous scale } 0 \text { [don't care either way] - } 100 \text { [extremely } \\
\text { strong preference] }\end{array}$ \\
\hline 5 & $\begin{array}{l}\text { How likely is it that your preferred Presidential candidate will } \\
\text { win? }\end{array}$ & Continuous scale 0 [no chance] - 100 [definite win] \\
\hline 6 & $\begin{array}{l}\text { How likely is it that election fraud will play a significant role in } \\
\text { the election outcome? }\end{array}$ & Continuous scale 0 [not at all] - 100 [extremely] \\
\hline 7 & $\begin{array}{l}\text { In order to make sure you read the questions, please choose the } \\
\text { lowest value in this scale [attention check] }\end{array}$ & Continuous scale 0 [not at all] - 100 [extremely] \\
\hline 8 & $\begin{array}{l}\text { * Hypothetical Dem/Rep map shown * } \\
\text { If the final election outcome looks like the map above, how } \\
\text { likely do you think it is that election fraud played a significant } \\
\text { role in this outcome? }\end{array}$ & Continuous scale 0 [not at all] - 100 [extremely] \\
\hline 9 & What is your age (in years)? (this question is optional) & Short open-ended text \\
\hline 10 & In which state do you currently reside? (this question is optional) & Dropdown list with all states \\
\hline
\end{tabular}

\section{Survey questions from the original survey.}




\section{Table S2.}

\begin{tabular}{lccc}
\hline Characteristic & Beta & $95 \% \mathbf{C l}^{1}$ & p-value \\
\hline (Intercept) & 0.45 & $0.39,0.51$ & $<0.001$ \\
\hline Prior fraud belief & -0.31 & $-0.39,-0.24$ & $<0.001$ \\
\hline Preferred candidate & & & \\
\hline Dem & 0.00 & $-0.09,0.08$ & $>0.9$ \\
\hline Rep & - & - & \\
\hline Preference strength & 0.10 & $0.04,0.15$ & 0.001 \\
\hline Prior win belief & 0.16 & $0.09,0.23$ & $<0.001$ \\
\hline Age & -0.08 & $-0.14,-0.02$ & 0.012 \\
\hline Survey submission order & -0.02 & $-0.08,0.04$ & 0.5 \\
\hline${ }^{1} \mathrm{Cl}=$ Confidence Interval & & & \\
\hline
\end{tabular}

Summary of multiple regression analysis, predicting the update of fraud belief following a losing map (posterior minus prior fraud belief, $\mathrm{z}$-scored; $\mathrm{N}=824$ ). Preferred candidate: Rep $=$ Trump, Dem $=$ Biden, a factor with contrasts coded as $\mathrm{Rep}=-1$ and Dem $=1$. All other variables are $\mathrm{z}-$ scored. 


\section{Table S3.}

\begin{tabular}{lccc}
\hline Characteristic & Beta & $95 \% \mathrm{Cl}^{1}$ & p-value \\
\hline Intercept) & -0.46 & $-0.50,-0.41$ & $<0.001$ \\
\hline Prior fraud belief & -0.60 & $-0.66,-0.55$ & $<0.001$ \\
\hline Preferred candidate & & & \\
\hline Dem & 0.01 & $-0.06,0.07$ & 0.8 \\
\hline Rep & - & - & \\
\hline Preference strength & -0.11 & $-0.16,-0.07$ & $<0.001$ \\
\hline Prior win belief & -0.03 & $-0.08,0.02$ & 0.3 \\
\hline Age & -0.03 & $-0.07,0.01$ & 0.2 \\
\hline Survey submission order & 0.00 & $-0.04,0.04$ & $>0.9$ \\
\hline${ }^{1} \mathrm{Cl}=$ Confidence Interval & & & \\
\hline
\end{tabular}

Summary of multiple regression analysis, predicting the update of fraud belief following a winning map (posterior minus prior fraud belief, z-scored; $\mathrm{N}=818$ ). Preferred candidate: $\mathrm{Rep}=$ Trump, Dem $=$ Biden, a factor with contrasts coded as Rep $=-1$ and Dem $=1$. All other variables are $\mathrm{z}$-scored. 
Table S4. 


\begin{tabular}{|c|c|c|}
\hline Order & Question & Answer structure \\
\hline 1 & What is your political affiliation? & Republican / Democrat / Independent / Other (explain) \\
\hline 2 & Did you vote in the 2020 presidential election last November? & Yes / No / Prefer not to say \\
\hline 3 & $\begin{array}{l}\text { Who was your preferred presidential candidate on election day } \\
\text { (November } 3,2020) \text { ? }\end{array}$ & Donald Trump / Joe Biden / Other \\
\hline 4 & How strong was your preference? & Continuous scale 0 [not at all] - 100 [extremely strong] \\
\hline 5 & Who is your preferred presidential candidate now? & Donald Trump / Joe Biden / Other \\
\hline 6 & How strong is your preference now? & Continuous scale 0 [not at all] - 100 [extremely strong] \\
\hline 7 & $\begin{array}{l}\text { Who won the } 2020 \text { election based on the vote count as certified } \\
\text { by the individual states? }\end{array}$ & Donald Trump / Joe Biden / Don't know \\
\hline 8 & How confident are you in your last answer? & Continuous scale 0 [not at all] - 100 [extremely] \\
\hline 9 & $\begin{array}{l}\text { Who won the } 2020 \text { election based on the electoral college vote } \\
\text { certified by Congress on January } 6 \text {, } \\
2021 ?\end{array}$ & Donald Trump / Joe Biden / Don't know \\
\hline 10 & How confident are you in your last answer? & Continuous scale 0 [not at all] - 100 [extremely] \\
\hline 11 & $\begin{array}{l}\text { Who would have won in a fraudless election? (i.e., one where } \\
\text { every vote reflects the true will of the American people) }\end{array}$ & Donald Trump / Joe Biden / Don't know \\
\hline 12 & How confident are you in your last answer? & Continuous scale 0 [not at all] - 100 [extremely] \\
\hline 13 & $\begin{array}{l}\text { What was the popular vote percentage in favor of Trump vs. } \\
\text { Biden as reported in state-reported vote counts (on average } \\
\text { across all states)? }\end{array}$ & $\begin{array}{l}\text { Continuous scale } 0[100 \% \text { Trump] - } 50 \text { [Even split } \\
(50 \% / 50 \%)]-100[100 \% \text { Biden] }\end{array}$ \\
\hline 14 & $\begin{array}{l}\text { What would the popular vote percentage in favor of Trump vs. } \\
\text { Biden have been in the absence of any fraud? }\end{array}$ & $\begin{array}{l}\text { Continuous scale } 0[100 \% \text { Trump }]-50 \text { [Even split } \\
(50 \% / 50 \%)]-100[100 \% \text { Biden] }\end{array}$ \\
\hline 15 & Who will be the U.S. President on January 21, $2021 ?$ & Donald Trump / Joe Biden / Other \\
\hline
\end{tabular}




\begin{tabular}{|c|c|c|}
\hline 16 & How confident are you in your last answer? & Continuous scale 0 [not at all] - 100 [extremely] \\
\hline 17 & $\begin{array}{l}\text { In order to make sure you are reading the questions, please } \\
\text { choose the highest value in this scale }\end{array}$ & Continuous scale 0 [not at all] - 100 [extremely] \\
\hline 18 & $\begin{array}{l}\text { How likely is it that election fraud affected the results in a } \\
\text { significant way in favor of: } \\
\text { * Joe Biden } \\
\text { * Donald Trump } \\
\text { (Order [Biden, Trump or Trump, Biden] counterbalanced across } \\
\text { participants) }\end{array}$ & Extremely unlikely / Unlikely / Likely / Extremely likely \\
\hline 19 & $\begin{array}{l}\text { What percentage of the official vote tally do you think was } \\
\text { fraudulent? }\end{array}$ & Continuous scale $0[<0.01 \%]-100[100 \%]$ \\
\hline 20 & Who benefited most from fraudulent voting activity? & $\begin{array}{l}\text { Continuous scale* } 0[100 \% \text { Trump }]-50 \text { [Even split } \\
(50 \% / 50 \%)]-100[100 \% \text { Biden] } \\
\text { * For half of the participants the order was reversed ( } 0 \text { being } \\
100 \% \text { Biden and } 100 \text { being } 100 \% \text { Trump) }\end{array}$ \\
\hline 21 & $\begin{array}{l}\text { Which of the following activities did you have in mind when } \\
\text { you answered the questions about fraud? (please check all that } \\
\text { apply) }\end{array}$ & $\begin{array}{l}\text { A list with a check box next to each of the following: } \\
\text { * Electorate manipulation prior to election day } \\
\text { * Vote buying / voter impersonation / misuse of proxy votes } \\
\text { * Ballot manipulation or ballot-box stuffing } \\
\text { * Mail-in ballot fraud } \\
\text { * Tampering with electronic voting systems } \\
\text { * Mis-recording of votes by state vote-counters and state } \\
\text { electoral officials } \\
\text { * Mis-reporting of final vote counts by state electoral officials } \\
\text { * Social media campaigns spreading false information } \\
\text { * False news stories by "right-wing" media } \\
\text { * False news stories by "left-wing" media } \\
\text { * False news stories by news aggregator services (e.g., } \\
\text { Reuters, Allied Press) } \\
\text { * Other (please explain) }\end{array}$ \\
\hline 22 & $\begin{array}{l}\text { Which of the following do you believe significantly impacted } \\
\text { the election results? }\end{array}$ & $\begin{array}{l}\text { Continuous scale } 0 \text { [No impact] - } 100 \text { [Extremely impactful] } \\
\text { for each of the above options }\end{array}$ \\
\hline 23 & $\begin{array}{l}\text { Who do you believe benefited most from each of the following } \\
\text { activities? }\end{array}$ & $\begin{array}{l}\text { Continuous scale* } 0[100 \% \text { Trump }]-50[50 \% / 50 \% \text { equal } \\
\text { benefit }]-100[100 \% \text { Biden }] \text { for each of the above options } \\
\text { * For half of the participant the order was reversed ( } 0 \text { being } \\
100 \% \text { Biden and } 100 \text { being } 100 \% \text { Trump) }\end{array}$ \\
\hline 24 & $\begin{array}{l}\text { Are you a U.S. Citizen or permanent resident? (this question is } \\
\text { optional) }\end{array}$ & Yes / No (participants answering "no" were excluded) \\
\hline 25 & What is your gender? (this question is optional) & Female / Male / Other / Prefer not to say \\
\hline 26 & What is your age (in years)? (this question is optional) & Short open-ended text \\
\hline
\end{tabular}




\begin{tabular}{|l|l|l|}
\hline 27 & In which state do you currently reside? (this question is optional) & Dropdown list with all states \\
\hline
\end{tabular}

Survey questions from the follow-up survey. 


\section{Table S5.}

\begin{tabular}{lccc}
\hline Characteristic & Beta & $95 \% \mathbf{C l}^{1}$ & p-value \\
\hline Intercept) & 0.19 & $0.15,0.23$ & $<0.001$ \\
\hline Prior win belief & 0.01 & $-0.04,0.05$ & 0.7 \\
\hline Preferred candidate & & & \\
\hline Dem & -0.67 & $-0.72,-0.63$ & $<0.001$ \\
\hline Rep & - & - & \\
\hline Preference strength & 0.09 & $0.04,0.13$ & $<0.001$ \\
\hline Age & -0.06 & $-0.10,-0.03$ & 0.001 \\
\hline Survey submission order & 0.00 & $-0.03,0.04$ & 0.8 \\
\hline Prior win belief * Preference strength & 0.02 & $-0.02,0.07$ & 0.4 \\
\hline Preferred candidate * Preference strength & & & \\
\hline Dem * Preference strength & -0.24 & $-0.29,-0.19$ & $<0.001$ \\
\hline${ }^{1}$ Cl = Confidence Interval & & & \\
\hline
\end{tabular}

Summary of multiple regression analysis, predicting the prior fraud belief ( $\mathrm{z}$-scored, $\mathrm{N}=1642$ ). Preferred candidate: Rep $=$ Trump, Dem $=$ Biden, a factor with contrasts coded as Rep $=-1$ and Dem $=1$. All other variables are $\mathrm{z}$-scored. 


\section{Table S6.}

\begin{tabular}{lccc}
\hline Characteristic & Beta & $95 \% \mathrm{Cl}^{1}$ & p-value \\
\hline (Intercept) & -0.44 & $-0.49,-0.39$ & $<0.001$ \\
\hline Prior win belief & -0.12 & $-0.19,-0.06$ & $<0.001$ \\
\hline Preference strength & -0.14 & $-0.19,-0.09$ & $<0.001$ \\
\hline Age & -0.09 & $-0.13,-0.04$ & $<0.001$ \\
\hline Survey submission order & -0.01 & $-0.06,0.03$ & 0.5 \\
\hline Prior win belief * Preference strength & 0.06 & $0.00,0.12$ & 0.063 \\
\hline${ }^{1} \mathrm{Cl}=$ Confidence Interval & & & \\
\hline
\end{tabular}

Summary of multiple regression analysis, predicting the prior fraud belief for Democrats (zscored, $\mathrm{N}=1032$ ). All variables are $\mathrm{z}$-scored. 
Table S7.

\begin{tabular}{lccc}
\hline Characteristic & Beta & $95 \% \mathrm{Cl}^{1}$ & p-value \\
\hline (Intercept) & 0.92 & $0.83,1.0$ & $<0.001$ \\
\hline Prior win belief & 0.10 & $0.03,0.17$ & 0.004 \\
\hline Preference strength & 0.23 & $0.12,0.35$ & $<0.001$ \\
\hline Age & -0.01 & $-0.08,0.06$ & 0.7 \\
\hline Survey submission order & 0.04 & $-0.03,0.11$ & 0.2 \\
\hline Prior win belief * Preference strength & -0.03 & $-0.10,0.05$ & 0.5 \\
\hline${ }^{1} \mathrm{Cl}=$ Confidence Interval & & & \\
\hline
\end{tabular}

Summary of multiple regression analysis, predicting the prior fraud belief for Republicans (zscored, $\mathrm{N}=610$ ). All variables are $\mathrm{z}$-scored. 
Table S8.

\begin{tabular}{lccc}
\hline Characteristic & Beta & $95 \% \mathrm{Cl}^{1}$ & $\mathrm{p}$-value \\
\hline Intercept) & 0.39 & $0.28,0.51$ & $<0.001$ \\
\hline Prior fraud belief & -0.38 & $-0.49,-0.26$ & $<0.001$ \\
\hline Preference strength & 0.03 & $-0.06,0.12$ & 0.6 \\
\hline Prior win belief & 0.23 & $0.09,0.36$ & $\mathbf{0 . 0 0 1}$ \\
\hline Age & -0.09 & $-0.18,-0.01$ & $\mathbf{0 . 0 3 3}$ \\
\hline Survey submission order & -0.02 & $-0.10,0.07$ & 0.7 \\
\hline${ }^{1} \mathrm{Cl}=$ Confidence Interval & & & \\
\hline
\end{tabular}

Summary of multiple regression analysis, predicting the update of fraud belief following a losing map for Democrats (posterior minus prior fraud belief, z-scored; $\mathrm{N}=516$ ). All variables are $\mathrm{z}$ scored. 


\section{Table S9.}

\begin{tabular}{lccc} 
Characteristic & Beta & $95 \% \mathbf{C l}^{1}$ & $\mathbf{p}$-value \\
\hline Intercept) & -0.41 & $-0.46,-0.35$ & $<0.001$ \\
\hline Prior fraud belief & -0.51 & $-0.57,-0.45$ & $<0.001$ \\
\hline Preference strength & -0.07 & $-0.11,-0.04$ & $<0.001$ \\
\hline Prior win belief & -0.02 & $-0.07,0.04$ & 0.5 \\
\hline Age & -0.02 & $-0.05,0.02$ & 0.4 \\
\hline Survey submission order & 0.00 & $-0.03,0.04$ & 0.9 \\
\hline${ }^{{ }^{1} \mathrm{Cl}=\text { Confidence Interval }}$ & & & \\
\hline
\end{tabular}

Summary of multiple regression analysis, predicting the update of fraud belief following a winning map for Democrats (posterior minus prior fraud belief, $\mathrm{z}$-scored; $\mathrm{N}=516$ ). All variables are z-scored. 
Table S10.

\begin{tabular}{lccc}
\hline Characteristic & Beta & $\mathbf{9 5 \%} \mathbf{C l}^{1}$ & $\mathbf{p}$-value \\
\hline Intercept) & 0.39 & $0.29,0.50$ & $<0.001$ \\
\hline Prior fraud belief & -0.27 & $-0.35,-0.20$ & $<0.001$ \\
\hline Preference strength & 0.18 & $0.11,0.24$ & $<0.001$ \\
\hline Prior win belief & 0.09 & $0.03,0.16$ & 0.005 \\
\hline Age & -0.06 & $-0.13,0.01$ & 0.084 \\
\hline Survey submission order & -0.03 & $-0.10,0.03$ & 0.3 \\
\hline${ }^{1} \mathrm{Cl}=$ Confidence Interval & & & \\
\hline
\end{tabular}

Summary of multiple regression analysis, predicting the update of fraud belief following a losing map for Republicans (posterior minus prior fraud belief, $\mathrm{z}$-scored; $\mathrm{N}=308$ ). All variables are $\mathrm{z}$ scored. 


\section{Table S11.}

\begin{tabular}{lrcc}
\hline Characteristic & Beta & $95 \% \mathrm{Cl}^{1}$ & p-value \\
\hline Intercept) & -0.40 & $-0.56,-0.25$ & $<0.001$ \\
\hline Prior fraud belief & -0.66 & $-0.77,-0.54$ & $<0.001$ \\
\hline Preference strength & -0.15 & $-0.26,-0.04$ & $\mathbf{0 . 0 0 8}$ \\
\hline Prior win belief & -0.02 & $-0.11,0.07$ & 0.7 \\
\hline Age & -0.05 & $-0.14,0.05$ & 0.3 \\
\hline Survey submission order & -0.01 & $-0.10,0.09$ & 0.9 \\
\hline${ }^{1} \mathrm{Cl}=$ Confidence Interval & & & \\
\hline
\end{tabular}

Summary of multiple regression analysis, predicting the update of fraud belief following a winning map for Republicans (posterior minus prior fraud belief, $\mathrm{z}$-scored; $\mathrm{N}=302$ ). All variables are z-scored. 


\section{Table S12.}

\begin{tabular}{|c|c|c|c|}
\hline Characteristic & $\log (\mathrm{OR})^{1}$ & $95 \% \mathrm{Cl}^{1}$ & p-value \\
\hline \multicolumn{4}{|l|}{ Fraud beneficiary } \\
\hline Joe Biden & 0.72 & $0.61,0.83$ & $<0.001$ \\
\hline Donald Trump & - & - & \\
\hline \multicolumn{4}{|l|}{ Preferred candidate } \\
\hline Dem & -1.1 & $-1.2,-1.0$ & $<0.001$ \\
\hline $\operatorname{Rep}$ & - & - & \\
\hline \multicolumn{4}{|c|}{ Fraud beneficiary * Preferred candidate } \\
\hline Joe Biden * Dem & -1.0 & $-1.1,-0.85$ & $<0.001$ \\
\hline
\end{tabular}

Summary of ordinal logistic regression, predicting the subjective probability of fraud benefiting each candidate $(N=937)$. Preferred candidate: the preferred candidate at the time of the followup survey $(\operatorname{Rep}=$ Trump, Dem $=$ Biden, a factor with contrasts coded as Rep $=-1$ and Dem $=1)$. Fraud beneficiary: the candidate for whom the probability of fraud is being considered (a factor with contrasts coded as Trump $=-1$ and Biden $=1$ ). 